\title{
Computational Design of Thermoelectric Alloys Through Optimization of Transport and Dopability
}

\begin{abstract}
Jiaxing Qu, ${ }^{a}$ Adam Balvanz, ${ }^{b}$ Sviatoslav Baranets,${ }^{b}$ Svilen Bobev, ${ }^{b}$ Prashun Goraic*
Alloying is a common technique to optimize the functional properties of materials for thermoelectrics, photovoltaics, energy storage etc. Designing thermoelectric (TE) alloys is especially challenging because it is a multi-property optimization problem, where the properties that contribute to high TE performance are interdependent. In this work, we develop a computational framework that combines first-principles calculations with alloy and point defect modeling to identify alloy compositions that optimize the electronic, thermal, and defect properties. We apply this framework to design $n$-type $\mathrm{Ba}_{2(1-x)} \mathrm{Sr}_{2 x} \mathrm{CdP}_{2}$ Zintl thermoelectric alloys. Our predictions of the crystallographic properties such as lattice parameters and site disorder are validated with experiments. To optimize the conduction band electronic structure, we perform band unfolding to sketch the effective band structures of alloys and find a range of compositions that facilitate band convergence and minimize alloy scattering of electrons. We assess the $n$-type dopability of the alloys by extending the standard approach for computing point defect energetics in ordered structures. Through the application of this framework, we identify an optimal alloy composition range with the desired electronic and thermal transport properties, and $n$-type dopability. Such a computational framework can also be used to design alloys for other functional applications beyond TE.
\end{abstract}

\section{Introduction}

Alloys are disordered solid solutions of two or more materials (metals or insulators), which could be iso- or hetero-structural. Such solid solutions may span the entire composition range (complete solid solutions) or may form in limited composition ranges with miscibility gap(s). Alloying has been utilized to design materials with superior structural and functional properties since time immemorial. From alloying copper with tin to improve the mechanical strength of tools and weaponry in the Bronze age to alloying of iron to produce steel during the Industrial Revolution, alloy design and optimization has become the cornerstone of human advancement. More recently, $\mathrm{In}_{x} \mathrm{Ga}_{1-x} \mathrm{~N}$ alloys have enabled tunable light emitting diodes, wherein the band gap of GaN is tuned by controlling the In content ${ }^{1}$. Alloying is extensively used to optimize functional properties of materials for photovoltaics (e.g., $\mathrm{CdSe}_{x} \mathrm{Te}_{1-x},{ }^{2} \mathrm{CsSn}_{x} \mathrm{~Pb}_{1-x} \mathrm{I}_{3}$ hybrid perovskites ${ }^{3}$ ), energy storage (e.g., $\mathrm{Li}_{6} \mathrm{PS}_{5-x} \mathrm{Se}_{x} \mathrm{Br}$ argyrodite solid electrolytes ${ }^{4}$ ), ferroelectrics (e.g., $\mathrm{Sc}_{x} \mathrm{Al}_{1-x} \mathrm{~N}^{5}$ ), and thermoelectrics (e.g., $\mathrm{PbSe}_{x} \mathrm{Te}_{1-x}{ }^{6}$ ) etc.

Unlike ordered and stoichiometric compounds, alloys represent a continuous compositional space and structural disorder adds to the complexity. Designing alloys to optimize functional properties in this continuous chemical space while also accounting for the effects of structural disorder is a formidable task. Empirical approaches have been quite successful for alloy design, especially high-throughput combinatorial thin film synthesis tech-

a University of Illinois at Urbana-Champaign, Urbana, IL 61801. ${ }^{b}$ University of Delaware, Newark, DE 19716. ' Colorado School of Mines, Golden, CO 80401. ${ }^{*} E-$ mail: pgorai@mines.edu

¿lectronic Supplementary Information (ESI) available niques. ${ }^{7}$ However, applications such as thermoelectrics that require synthesis of bulk (vs. thin-film) samples, alloy optimization remains a tedious experimental process relying on trial-and-error and chemical intuition.

Designing thermoelectric (TE) alloys is particularly challenging because it is a multi-property optimization problem, where the properties that contribute to high TE performance are interdependent. Alloying has been employed to optimize a variety of properties that directly or indirectly affect the TE performance: (1) enhance phonon scattering to reduce lattice thermal conductivity, ${ }^{8,9}$ (2) promote band convergence to improve power factor, ${ }^{8,10-12}$ (3) tune band gap to suppress bipolar conduction, ${ }^{8,10,11}$ and (4) optimize dopability ${ }^{13}$. Figure 1 summarizes some of the beneficial effects of alloying on the TE performance, including enhanced power factor through band convergence, optimized doping by manipulating the native defects, and reduced lattice thermal conductivity due to increased phonon scattering and lattice softening. Notably, automated high-throughput experimental procedures are being developed to synthesize bulk samples with the goal of discovering new TE materials and optimizing TE alloys. ${ }^{14,15}$

Computations have greatly facilitated new TE materials discovery, ${ }^{16-19}$ and their optimization through electronic doping ${ }^{13,20}$. At the same time, first-principles modeling of TE alloys has provided useful mechanistic insights ${ }^{10,21}$; however, a systematic computational framework for designing and optimizing TE alloys has not been demonstrated yet. In concert with highthroughput bulk synthesis, computations can provide guidance to navigate the continuous compositional space, and identify the optimal compositions in the multi-property phase space. Outside of thermoelectrics, computations have been successfully em- 


\section{Enhanced $S^{2} \sigma$ through} band convergence
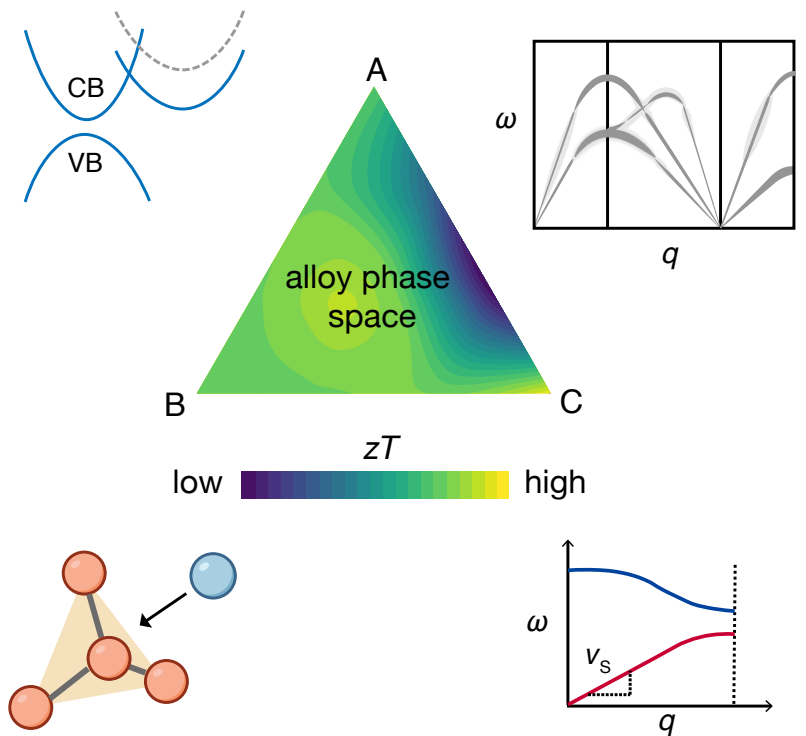

Improved dopability due changes in defect chemistry
Reduced $\kappa_{L}$ due to phonon scattering

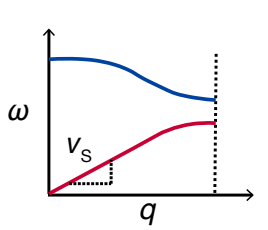

Reduced $\kappa_{\mathrm{L}}$ due to phonon scattering
Fig. 1 Beneficial effects of alloying on the electronic and thermal transport and doping of thermoelectric materials. Band convergence leads to higher power factor $\left(S^{2} \sigma\right)$, increased phonon scattering and lattice softening lowers lattice thermal conductivity $\left(\kappa_{L}\right)$, and changes in defect chemistry may promote dopability.

ployed to design high-entropy ${ }^{22-24}$ and functionally-graded alloys ${ }^{25,26}$, suggesting that it is possible to develop a combined theory-experiment framework for rational design and optimization of TE alloys.

In this work, we develop a computational framework for designing TE alloys by combining first-principles calculations with alloy and point defect modeling. We believe that this is the first computational work within thermoelectrics that simultaneously considers the effects of alloying on the transport properties and doping. We apply this framework to identify specific compositions of $n$-type $\mathrm{Ba}_{2(1-x)} \mathrm{Sr}_{2 x} \mathrm{CdP}_{2}$ Zintl alloys that optimize the electronic and phonon transport properties, as well as $n$ type dopability. The computational framework builds upon wellestablished alloy modeling techniques, including special quasirandom structures (SQS) ${ }^{27}$ and effective band structure (EBS) ${ }^{28}$ to quantify the effect of alloying on the transport properties. To assess the dopability of alloys, we extend a standard approach for computing point defect energetics ${ }^{29,30}$ in ordered structures, akin to our prior work on defect modeling of complex disordered structures. ${ }^{31}$ Through the application of this computational framework, we identify $\mathrm{Ba}_{2(1-x)} \mathrm{Sr}_{2 x} \mathrm{CdP}_{2}$ alloy compositions that minimize alloy scattering of electrons, promote conduction band convergence, lower lattice thermal conductivity and are $n$-type dopable. We validate the predicted crystallographic and disorder properties by synthesizing and characterizing a series of alloy compositions.

\section{Results and Discussion}

We demonstrate the application of the computational alloy optimization framework by identifying $\mathrm{Ba}_{2(1-x)} \mathrm{Sr}_{2 x} \mathrm{CdP}_{2}$ Zintl phase alloys that simultaneously achieve: (1) conduction band convergence, (2) reduced alloy scattering of electrons, (3) reduced lattice thermal conductivity, and (4) suitable $n$-type dopability. As schematically illustrated in Figure 1, band convergence enhances the power factor while reduced alloy scattering of electrons minimizes reduction of the intrinsic carrier mobility. A reduction in the lattice thermal conductivity is expected due to increased phonon scattering and lattice softening. Finally, it has to be examined how alloying affects $n$-type dopability. The details of the firstprinciples calculations, alloy models, and defect calculations can be found in Section 4.

\subsection{Crystal Structure and Site Selectivity}

Zintl phases $A_{2} \mathrm{CdP}_{2}(A=\mathrm{Sr}, \mathrm{Ba})$ crystallize in a noncentrosymmetric orthorhombic space group ( $C m c 2_{1}$, no. 36). There are 5 unique atomic positions, all of which are $4 a$ Wyckoff sites (site symmetry $m$ ). The structures belong to a family of compounds, ${ }^{9,30,32,33}$ which crystallize in the well-established $\mathrm{Yb}_{2} \mathrm{CdSb}_{2}$ structure type. ${ }^{34}$ This collection of compounds have been the subject of investigation in the past, including a detailed study ${ }^{30}$ conducted by our team on the parent compounds $\left(\mathrm{Sr}_{2} \mathrm{CdP}_{2}\right.$ and $\left.\mathrm{Ba}_{2} \mathrm{CdP}_{2}\right)$ of the Ba-Sr-Cd-P quaternaries that we presently discuss. These parent compounds feature a layered structure comprised of $\mathrm{CdP}_{4}$ tetrahedra, forming $\left[\mathrm{CdP}_{2}\right]^{4-}$ layers (Figure 2), with $\mathrm{Ba} / \mathrm{Sr}$ atoms occupying sites between the $\left[\mathrm{CdP}_{2}\right]^{4-}$ layers (interlayer site 1 ) and within the $\left[\mathrm{CdP}_{2}\right]^{4-}$ layers (intralayer site 2). There are an equal number of sites 1 and 2 available in the structure. Since $\mathrm{Ba}_{2(1-x)} \mathrm{Sr}_{2 x} \mathrm{CdP}_{2}$ are isostructural alloys with no observed changes in the layering and coordination, the reader is referred to our previous work ${ }^{30}$ for the overall structural description.

We model $\mathrm{Ba}_{2(1-x)} \mathrm{Sr}_{2 x} \mathrm{CdP}_{2}$ as random alloys using special quasirandom structures (SQS). The details of SQS generation and convergence tests are provided in the Methods (Section 4.2). The free energy of mixing $\left(\Delta G_{m}\right)$ of $\mathrm{Ba}_{2(1-x)} \mathrm{Sr}_{2 x} \mathrm{CdP}_{2}$ alloys is calculated from their DFT total energy and configurational entropy, as detailed in Section 4.3. We predict based on the computed $\Delta G_{m}$ that $\mathrm{Ba}_{2(1-x)} \mathrm{Sr}_{2 x} \mathrm{CdP}_{2}$ forms a complete solid solution i.e., $\mathrm{Sr}_{2} \mathrm{CdP}_{2}$ and $\mathrm{Ba}_{2} \mathrm{CdP}_{2}$ are soluble for all $x$. In Figure $3, \Delta G_{m}$ is negative for all values of $x$ suggesting the formation of a complete solid solution. More interestingly, our calculations reveal site selectivity of the disorder depending on whether the alloy composition is Ba-rich $(x<0.5)$ or Sr-rich $(x>0.5)$. Specifically, we find that for Ba-rich compositions $(x<0.5)$, it is energetically more favorable to fully occupy site 1 with $\mathrm{Ba}$ while confining $\mathrm{Ba} / \mathrm{Sr}$ disorder to site 2 as compared to fully occupying site 2 and confining disorder to site 1 . Disorder on both sites 1 and 2 is energetically even more unfavorable for all $x<0.5$ and $x>0.5$. Similarly, for Srrich compositions $(x>0.5)$, the full occupancy of site 2 by Sr and confining $\mathrm{Ba} / \mathrm{Sr}$ disorder to site 1 is energetically favorable. This site selectivity is a direct consequence of the relative ionic size of $\mathrm{Ba}$ and $\mathrm{Sr}$ and the space available in the interlayer and intralayer 


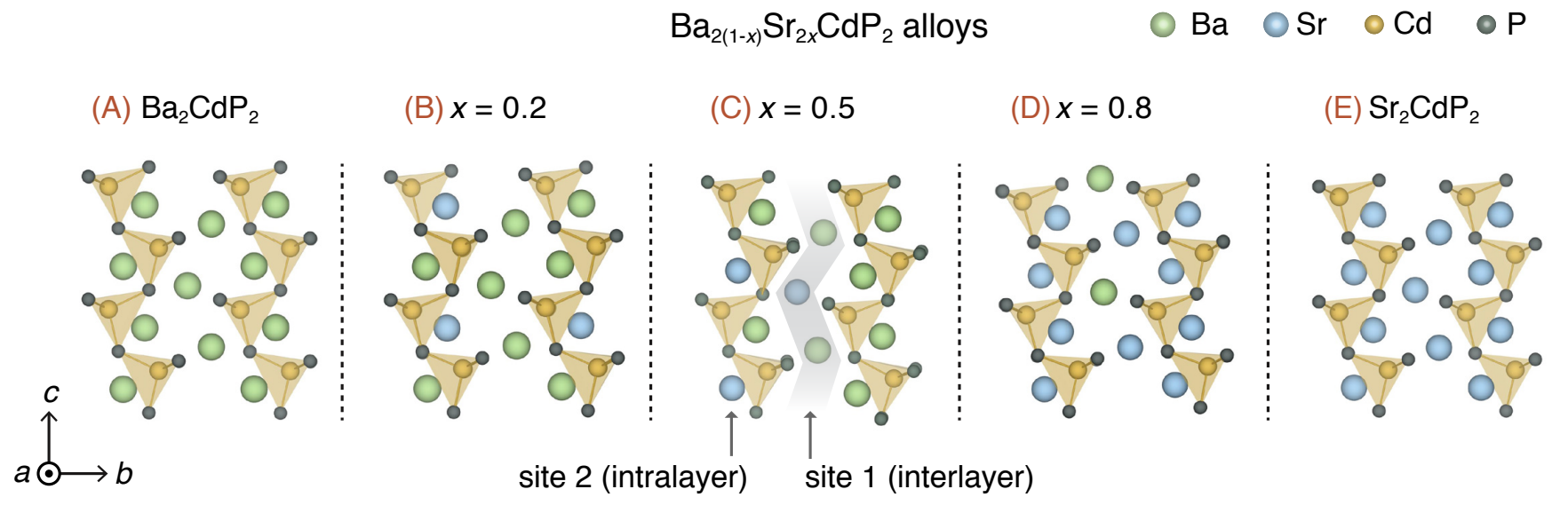

Fig. 2 Schematic representations of the crystal structures of $\mathrm{Ba}_{2(1-x)} \mathrm{Sr}_{2 x} \mathrm{CdP}_{2}$ alloys with five different compositions. The end members with $x=0$ and $x=1$ are the parent compounds $\mathrm{Ba}_{2} \mathrm{CdP}_{2}$ and $\mathrm{Sr}_{2} \mathrm{CdP}_{2}$, whose structures are depicted in $(\mathrm{A})$ and $(\mathrm{E})$, respectively. Ba-rich $(x=0.2)$ and $\mathrm{Sr}$-rich $(x$ $=0.8$ ) compositions are shown in (B) and (D), respectively. Illustrative drawing of the structure of the 50-50 alloy is represented in (C). Ba and Sr occupy two different Wyckoff sites, with site 1 located between the layers (interlayer) and site 2 within the layers (intralayer). In Ba-rich compositions, $\mathrm{Ba}$ atoms fully occupy interlayer site 1 and disorder is observed at site 2 . The intralayer site 2 is fully occupied by $\mathrm{Sr}$ in $\mathrm{Sr}$-rich compositions with $\mathrm{Sr} / \mathrm{Ba}$ disorder on site 1.

sites of the layered structure of $\mathrm{Ba}_{2(1-x)} \mathrm{Sr}_{2 x} \mathrm{CdP}_{2}$. Figure 2 shows the site selectivity for representative Ba-rich $(x=0.2)$ and Sr-rich $(x=0.8)$ compositions.

The 50-50 alloy $(x=0.5)$ is unique in that the $\mathrm{Ba} / \mathrm{Sr}$ disorder must occur on both sites 1 and 2 because they are present in equal numbers in the structure. The site selectivity of $\mathrm{Ba}$ and Sr cannot be simultaneously accommodated for $x=0.5$. Our calculated $\Delta G_{m}$ (Figure 3) suggests that it is possible to form the 50-50 alloy $\left(\Delta G_{m}<0\right)$ but energetically much less stable than compositions with $x=0.5-\delta$ and $x=0.5+\delta$, where $\delta$ is a small positive number. As such, we predict that it will be challenging to experimentally synthesize the exact 50-50 alloy. To validate

\begin{tabular}{lllllll}
\hline$x$ & $\begin{array}{l}\text { Site } 1 \\
\mathrm{Ba} / \mathrm{Sr} \\
\text { ratio }\end{array}$ & $\begin{array}{l}\text { Site } 2 \\
\mathrm{Ba} / \mathrm{Sr} \\
\text { ratio }\end{array}$ & $\begin{array}{l}a \\
(\AA)\end{array}$ & $\begin{array}{l}b \\
(\AA)\end{array}$ & $\begin{array}{l}c \\
(\AA)\end{array}$ & $\begin{array}{l}V \\
\left(\AA^{3}\right)\end{array}$ \\
& & & & & & \\
\hline & & & & & & \\
0.00 & Ba only & Ba only & 4.47 & 17.20 & 7.92 & 603 \\
0.20 & Ba only & $0.57 / 0.43$ & 4.45 & 17.07 & 7.72 & 586 \\
0.35 & Ba only & $0.32 / 0.68$ & 4.44 & 17.10 & 7.59 & 575 \\
0.40 & Ba only & $0.19 / 0.81$ & 4.44 & 17.12 & 7.52 & 568 \\
0.45 & Ba only & $0.10 / 0.90$ & 4.44 & 17.15 & 7.47 & 567 \\
0.55 & $0.90 / 0.10$ & Sr only & 4.41 & 17.13 & 7.46 & 564 \\
0.65 & $0.70 / 0.30$ & Sr only & 4.39 & 17.01 & 7.44 & 556 \\
0.70 & $0.56 / 0.44$ & Sr only & 4.38 & 16.92 & 7.45 & 552 \\
0.80 & $0.41 / 0.59$ & Sr only & 4.37 & 16.80 & 7.45 & 547 \\
1.00 & Sr only & Sr only & 4.34 & 16.51 & 7.47 & 533 \\
& & & & & & \\
\hline
\end{tabular}

Table 1 Crystallographic and refinement parameters for the compositions of the solid solution $\mathrm{Ba}_{2(1-x)} \mathrm{Sr}_{2 x} \mathrm{CdP}_{2}$. For Ba-rich conditions $(x=0.00-0.45)$, a disorder site selectivity for site 2 is observed while for Sr-rich $(x=0.55-1.00)$ conditions, disorder prefers to occur at site 1. our predictions of site selectivity, we synthesized a series of alloy compositions (Section 4.6) ranging from $x=0\left(\mathrm{Ba}_{2} \mathrm{CdP}_{2}\right)$ to $x=1$ $\left(\mathrm{Sr}_{2} \mathrm{CdP}_{2}\right)$ and performed single-crystal XRD measurements (Section 4.7) to determine the crystallographic parameters, which are summarized in Table 1.

Table 1 summarizes the Ba and Sr occupancy of sites 1 and 2, the cell parameters $(a, b, c)$, and unit cell volume $(V)$ as a function of the alloy composition $x$. Our predicted site selectivity is confirmed by experiments. For Ba-rich alloys $(x<0.5)$, site 1 is

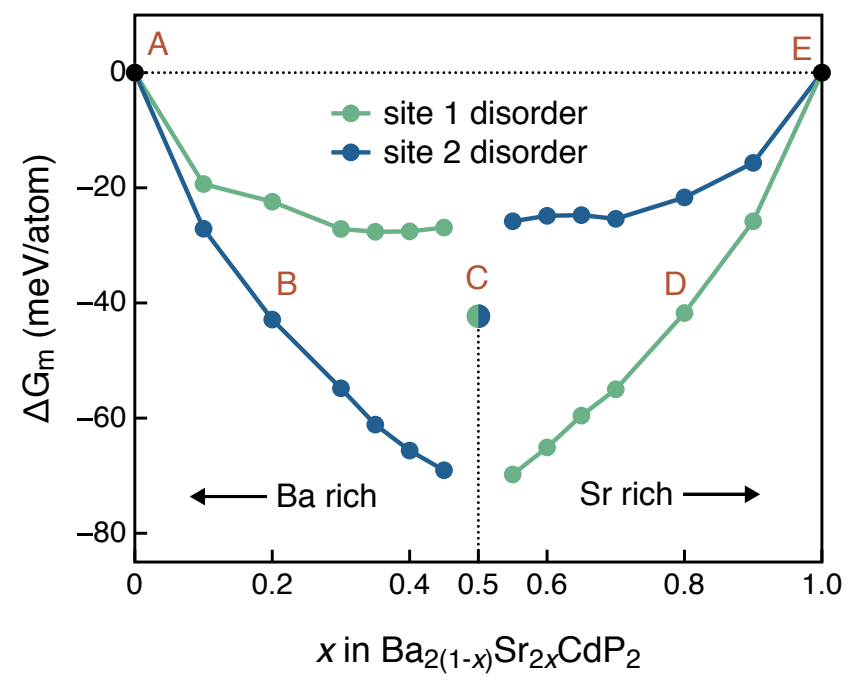

Fig. 3 The free energy of mixing $\left(\Delta G_{\mathrm{m}}\right)$ of $\mathrm{Ba}_{2(1-x)} \mathrm{Sr}_{2 x} \mathrm{CdP}_{2}$ alloys as a function of $x$ calculated at $900 \mathrm{~K}$, the typical synthesis temperature of these Zintl phases. Labels $(A)-(E)$ correspond to the crystal structures shown in Figure 2. For Ba-rich compositions $(x<0.5)$, it is more favorable for $\mathrm{Ba} / \mathrm{Sr}$ disorder to occur on the intralayer site 2. Conversely, the interlayer site 1 is disorder in Sr-rich compositions $(x>0.5)$. The 50-50 alloy is energetically less stable. 

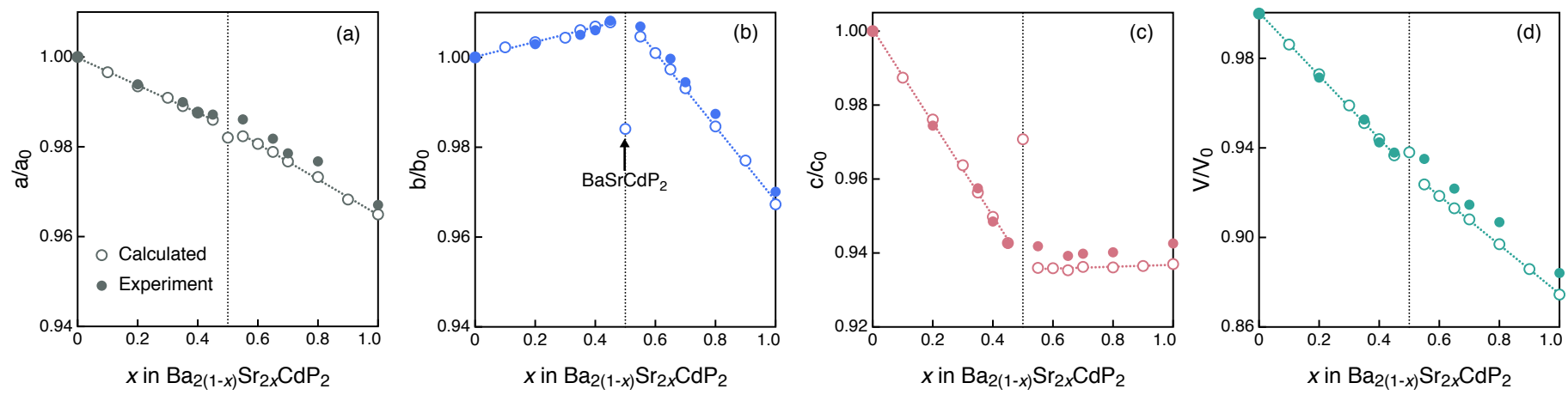

Fig. 4 Normalized lattice parameters $a, b$, and $c$ and unit cell volume $(V)$ of $\mathrm{Ba}_{2(1-x)} \mathrm{Sr}_{2 x} \mathrm{CdP}_{2}$ alloys as a function of $x$. Here, the calculated and experimental lattice parameters and volume are normalized by the values for $\mathrm{Ba}_{2} \mathrm{CdP}_{2}(x=0)$. Open markers are calculated and filled markers obtained from experimental measurements.

fully occupied by Ba i.e., no Ba/Sr disorder while for Sr-rich alloys $(x>0.5)$, site 2 is fully occupied by Sr. Experimentally, it has proven difficult to obtain structures with composition representing perfect $\mathrm{Ba}_{1.0} \mathrm{Sr}_{1.0} \mathrm{CdP}_{2}$. The computed lattice parameters and unit cell volume are in excellent agreement with experimental measurements, as shown in Figure 4. Both computed and experimental values in Figure 4 are normalized by the values for $\mathrm{Ba}_{2} \mathrm{CdP}_{2}(x=0)$. The good agreement between theory and experiments provides confidence in the $\mathrm{Ba}_{2(1-x)} \mathrm{Sr}_{2 x} \mathrm{CdP}_{2}$ alloy models created using special quasi-random structures (Section 4).

With increasing Sr content (increasing $x$ ), we observe a nearlinear decrease in the unit cell volume consistent with Vegard's law (Figure 4d). Similarly, the cell parameter $a$ exhibits an almost linear decrease with $x$, but cell parameters $b$ and $c$ change nonmonotonically (Figures $4 \mathrm{~b}, 4 \mathrm{c}$ ). Theoretically, we predict that the $50-50$ alloy $(x=0.5)$ represents an inflection point in $b$ and $c$ as a function of $x$, which is consistent with experiments. The nonmonotonic changes and inflection in $b$ and $c$ are due to the site selectivity of $\mathrm{Ba}$ and $\mathrm{Sr}$ and the related structural distortions, as discussed above.

The addition of small amounts of Ba, starting from the parent compound $\mathrm{Sr}_{2} \mathrm{CdP}_{2}(x=1)$, causes a sharp increase in $b$, which is also the layer stacking direction (Figure 2). This is explained by the fact that under Ba-poor conditions, Ba occupies the interlayer positions (site 1), a site preference which has also been observed in the $\mathrm{Sb}$ analogue of these quaternary structures ${ }^{32}$. This introduction of the larger $\mathrm{Ba}$ cation into the interlayer site (site 1) forces the $\left[\mathrm{CdP}_{2}\right]^{4-}$ polyanionic layers further apart from one another to accommodate the larger cation. The direct evidence for this expansion can be found in the increase in the Ba1/Sr1-P1/P2 bond lengths (Table S3), which represent the base of the square pyramidal coordination.

The sharp changes in $c$ can also be rationalized via the larger ionic radius of $\mathrm{Ba}$ when compared to Sr. Under Ba-rich conditions $(x<0.5)$ Ba enters site 2 , causing local distortions of the $\mathrm{CdP}_{4}$ tetrahedra, including and increase in the the P1-Cd-P1 the CdP1-Cd bond angles (Table S4). Such distortions of the layers are indicative of the structure accommodating a larger cation in site 2. These local distortions then lead to an overall increase in the global $c$ unit cell parameter.

\subsection{Optimization of Electronic Transport}

In the context of alloy optimization, there are two aspects of the electronic structure that affects carrier transport, namely, band convergence and alloy scattering. For thermoelectrics, convergence of light bands (low effective mass) is desirable to enhance the Seebeck coefficient and the power factor (Figure 1). In contrast, alloy scattering of electronic carriers (electrons, holes) is undesirable because it reduces carrier mobility and therefore, electronic conductivity. Our previous work ${ }^{30}$ has shown that $\mathrm{Sr}$ substitution on the interlayer site 1 (Figure 2) in $\mathrm{Ba}_{2} \mathrm{CdP}_{2}$ induces convergence of two conduction bands located at $\Gamma$ and $Z$ (Figure 5). Our goal in this work is to computationally identify the optimal $\mathrm{Ba}_{2(1-x)} \mathrm{Sr}_{2 x} \mathrm{CdP}_{2}$ alloy for $n$-type transport. As such, we focus on conduction band (CB) convergence and alloy scattering of electrons.

However, random substitutional alloys lack formal translational symmetry and therefore, cannot be described by the standard bandstructure $E(\vec{k})$ dispersion. We use the effective band structure (EBS), proposed by Popescu and Zunger, ${ }^{28}$ to interpret the conduction band electronic structure of the alloys. The computational details of EBS calculations are provided in the Methods (Section 4.4). The EBS is obtained by unfolding the SQS supercell bands to the primitive cell Brillouin zone using spectral decomposition. The resulting EBS reveals the extent to which the band characteristics are preserved or lost. Band convergence can be determined by examining the EBS. In addition, the linewidth or band broadening at each $E(\vec{k})$ is reflective of the electronic carrier lifetimes due to alloy scattering; the larger the band broadening (more "smeared" bands), the shorter the carrier lifetimes.

The computed EBS of $\mathrm{Ba}_{2(1-x)} \mathrm{Sr}_{2 x} \mathrm{CdP}_{2}$ alloys are shown for 12 different compositions in Figure 5, ranging from Ba-rich $(x=0.1)$ to Sr-rich $(x=0.9)$. We find that the relative position (on the energy axis) of the two low-lying CBs at $\Gamma$ and $Z$ changes with $x$. In the parent compounds, $\mathrm{Ba}_{2} \mathrm{CdP}_{2}$ and $\mathrm{Sr}_{2} \mathrm{CdP}_{2}$, the energy separation $\left(\Delta E_{\Gamma-Z}\right)$ between the two CBs are $0.26 \mathrm{eV}$ and $0.35 \mathrm{eV}$, respectively (Figure $6 \mathrm{a}$ ). The small $\Delta E_{\Gamma-Z}$ in the parent compounds provides an opportunity to achieve band convergence through al- 

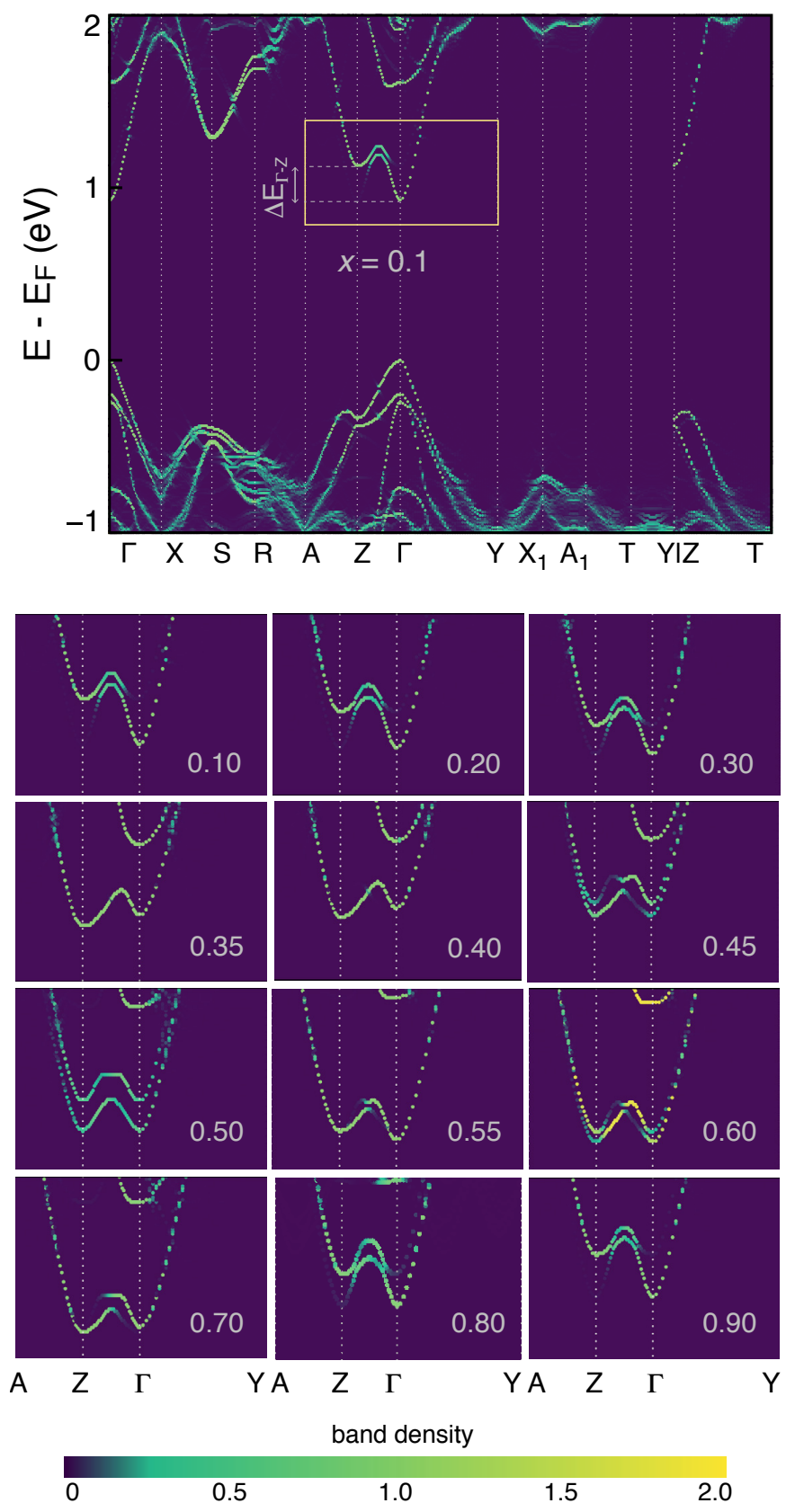

Fig. 5 The effective band structure (EBS) of $\mathrm{Ba}_{2(1-x)} \mathrm{Sr}_{2 x} \mathrm{CdP}_{2}$ alloys along special $\vec{k}$-point paths of the orthorhombic Brillouin zone for 12 different compositions, ranging from Ba-rich $(x=0.10)$ to Sr-rich $(x=0.90)$. The top panel shows the EBS for $x=0.1$ over a wider range of energy, where the two conduction bands at $\Gamma$ and $Z$ are highlighted by the inset box and the energy separation between the two bands $\left(\Delta E_{\Gamma-Z}\right)$ is $0.25 \mathrm{eV}$. The lower panels show the zoomed-in inset region of interest for a wide range of compositions $(x)$. The color scale represents the band density, which is the number of bands (in the alloy supercell) that are unfolded at a given primitive cell wave vector $\vec{k}$ and energy $E$.

loying. In the composition range $x=0.0-0.30$, the CB dispersion near $\Gamma$ and $Z$ closely resemble that of the parent compound, $\mathrm{Ba}_{2} \mathrm{CdP}_{2} . \Delta E_{\Gamma-Z}$ decreases with increasing Sr content, as seen in the regime R1 in Figure 6(a). In the regime R2, which spans $x=0.35-0.80$, we observe convergence of the CBs at $\Gamma$ and $Z$ within $30 \mathrm{meV}$. Interestingly, for $x=0.35$ and $x=0.40$, the conduction band minima is located at $Z$, resulting in indirect gap unlike the parents compounds and the other alloy compositions. The 5050 alloy $(x=0.5)$ is unique in that the two converged CBs have similar dispersion (Figure 5) making it a candidate for optimal composition. However, we know that the 50-50 alloy is expected to be thermodynamically less stable based on the free energy of mixing (Section 2.1). Finally, for compositions with $x=0.8-1.0$ in regime $\mathrm{R} 3$ (Figure $6 \mathrm{a}$ ), $\Delta E_{\Gamma-Z}$ again increases until it reaches $0.35 \mathrm{eV}$ for the parent compound, $\mathrm{Sr}_{2} \mathrm{CdP}_{2}$. In summary, $\mathrm{CB}$ convergence is achieved for compositions $x=0.35-0.70$ (regime $\mathrm{B}$ in Figure 6). We demonstrate with this example how band convergence can be determined through the calculation of EBS.

Next, we examine band broadening in the calculated EBS to assess the extent of alloy scattering of electronic carriers. We observe significant broadening (smearing) in the valence band beyond $\sim 0.5 \mathrm{eV}$ from the band edge (shown only for $x=0.1$ in Figure 5). Since we are interested in $n$-type transport, we focus on the broadening in the CB, particularly around the band edges at $\Gamma$ and $Z$. For compositions between $x=0.35-0.65$ (EBS for alloy composition $x=0.65$ is shown in Figure S2), we observe weak band broadening, which suggests that the electronic carrier mobility in this composition range will remain largely unaffected due to alloy (disorder) scattering. As such, alloy compositions $x=0.35-0.70$, which preserve the Bloch character of the CBs around $\Gamma$ and $Z$, are optimal for minimizing alloy scattering of carriers. In particular, for $x=0.35$ and $x=0.45$, the CBs near $\Gamma$ and $Z$ are almost unperturbed by the alloy disorder, for reasons that are not immediately clear to us. Fortuitously, the compositions where both band convergence is achieved and alloy scattering is minimized overlap.

\subsection{Optimization of Thermal Properties}

Alloying generally reduces lattice thermal conductivity $\left(\kappa_{L}\right)$ due to increased phonon scattering. Further reduction in $\kappa_{L}$ may result from lattice softening, as observed in SnTe-AgSbTe 2 alloys ${ }^{35}$ and Na-doped PbTe. ${ }^{36} \kappa_{L}$ can be directly determined from first principles by calculating the second and third-order interatomic force (IFCs) constants ${ }^{37}$. In these calculations, the effects of both phonon scattering and lattice softening are captured. However, in the finite-displacement method, the evaluation of the third-order IFCs requires pairwise atomic displacements to be performed in a chosen supercell, which rapidly scales with the supercell size. For alloys, where the "unit cell" is already a supercell, such calculations are prohibitively expensive. In contrast, the effect of alloying on lattice softening can be assessed from first principles by computing the speed of sound. In this work, we focus on lattice softening. Alloy scattering of phonons will only further reduce $\kappa_{L}$.

We computed the speed of sound $\left(v_{s}\right)$ of each alloy composition from the bulk modulus $(B)$ using the approximate relation $v_{s}=\sqrt{B / \rho}$, where $\rho$ is the density. ${ }^{38}$ Figure $6 \mathrm{~b}$ shows the variation of $v_{s}$ and $B$, both of which linearly increases with the alloy composition $x$ following Vegard's law. Replacing $\mathrm{Ba}$ in the parent compound, $\mathrm{Ba}_{2} \mathrm{CdP}_{2}$, with $\mathrm{Sr}$, which is less electropositive and has smaller atomic mass, makes the alloy stiffer (higher $B$ ). However, 

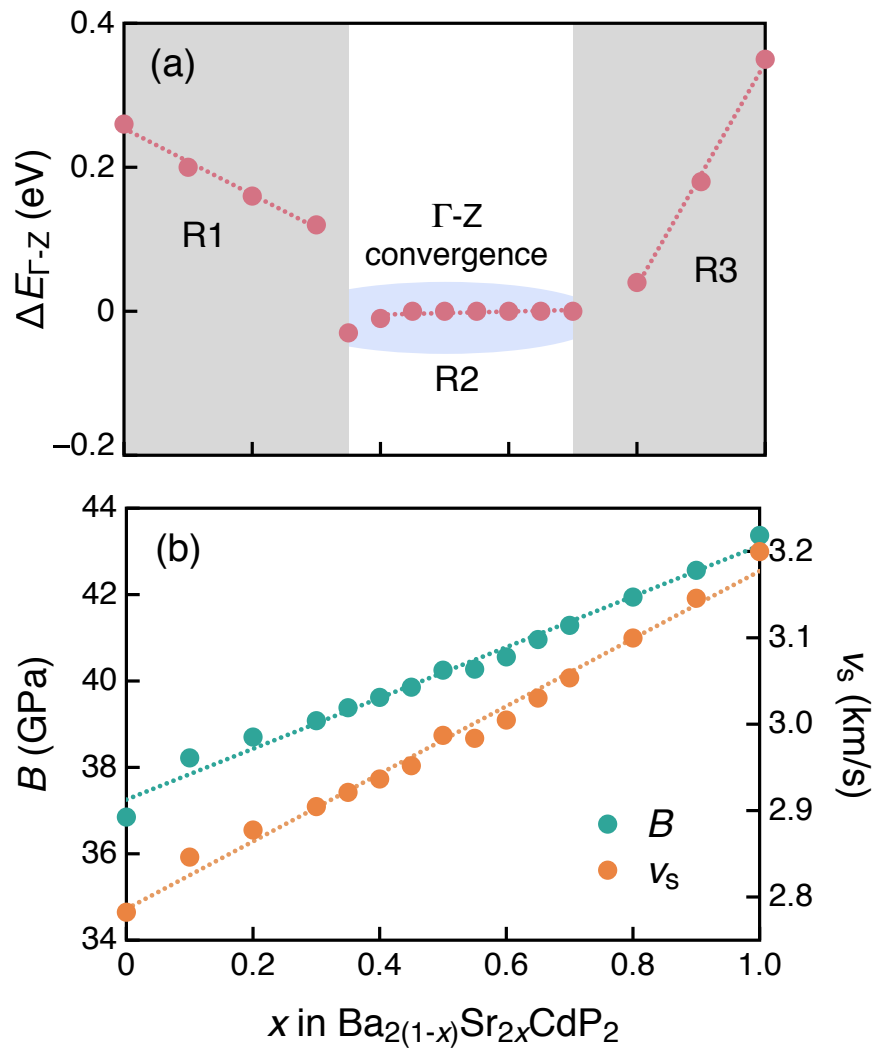

Fig. 6 (a) Energy separation $\left(\Delta E_{\Gamma-Z}\right)$ between conduction band edges at $\Gamma$ and $Z$ calculated from the effective band structures shown in Figure 5). (b) Calculated bulk modulus $(B)$ and speed of sound $\left(v_{s}\right)$ as a function of composition $x$ for $\mathrm{Ba}_{2(1-x)} \mathrm{Sr}_{2 x} \mathrm{CdP}_{2}$ alloys.

due to the small change in $B(<2 \mathrm{GPa})$ and $v_{s}(<0.15 \mathrm{~km} / \mathrm{s})$ between $x=0.35-0.70$, we do not expect a significant variation in $\kappa_{L}$ in this range due to lattice softening/stiffening. As a result, after considering electronic transport and $\kappa_{L}$, the optimal composition range is still $x=0.35-0.70$. Therefore, we choose one of the compositions within this range, $\mathrm{Ba}_{0.75} \mathrm{Sr}_{1.25} \mathrm{CdP}_{2}(x=0.625)$ to check if this optimal compositions is $n$-type dopable.

\subsection{Assessment of Alloy Dopability}

The thermoelectric performance of a material critically depends on whether it can be doped with the desired carrier type (electrons or holes). Furthermore, the carrier concentration of the desired carrier type needs to be tuned to optimize the TE performance. ${ }^{39}$ The presence of high concentrations of "killer" chargecompensating native defects can prevent the desired doping. For example, high concentrations of acceptor cation vacancies in $\mathrm{Mg}_{3} \mathrm{Sb}_{2}$ and BiCuOSe under cation-poor growth conditions, respectively, prevent $n$-type doping. ${ }^{13,40}$ In the context of alloy design, it is crucial that the compositions that optimize the electronic and thermal transport are also dopable.

A material is considered $n$-type dopable if the most favorable native acceptor defect has high formation energy. In such a case, electrons generated by a sufficiently soluble donor dopant are not compensated by the holes created by the native acceptor. In other words, a "killer" acceptor defect is absent under the chosen condi- tions. Accordingly, an $n$-type dopability window $\left(\Delta E_{n}\right)$ may be defined at the CBM (Figure 7), where a large positive $\Delta E_{n}$ indicates a highly $n$-type dopable material while a negative window would suggest difficulty in $n$-type doping. Similarly, a $p$-type dopability window $\left(\Delta E_{p}\right)$ is set by the formation energy of the favorable native donor defect at the VBM and provides a measure of the potential for $p$-type doping.

In this study, we are concerned with the $n$-type dopability of $\mathrm{Ba}_{2(1-x)} \mathrm{Sr}_{2 x} \mathrm{CdP}_{2}$ alloys. We chose to verify the $n$-type dopability of $\mathrm{Ba}_{0.75} \mathrm{Sr}_{1.25} \mathrm{CdP}_{2}(x=0.625)$, which lies in the optimal composition range of $x=0.35-0.70$ based on the electronic and thermal transport properties (Sections 2.2, 2.3). The periodic supercell approach utilized in first-principles calculations of defect energetics is well established for ordered and stoichiometric materials. ${ }^{13,29,41}$ A direct extension of the supercell approach to alloys is not straightforward because of the presence of different local environments in the disordered structures. As such, DFT relaxations of hundreds of supercells may be required to compute the defect formation energetics in alloys, which makes this approach prohibitively expensive. For example, in a 100-atom SQS supercell of $\mathrm{Ba}_{0.75} \mathrm{Sr}_{1.25} \mathrm{CdP}_{2}$, there are 25 unique $\mathrm{Sr}$ sites. Therefore, calculating the formation energy of $\mathrm{Sr}$ vacancies $\left(\mathrm{V}_{\mathrm{Sr}}\right)$ in a single charge state (typically, multiple charge states of each defect are calculated) amounts to 25 different DFT supercell relaxations. It quickly becomes apparent that considering all types of defects, including vacancies, anti-sites, and interstitials, in different charge states requires hundreds of DFT supercell calculations. Furthermore, in a disordered material, the different local environments can lead to variations in the formation energies of the same defect. Therefore, it is fundamentally important to determine the statistical variation in the defect formation energies.

We adapt the standard supercell approach to calculate the range of formation energies of each defect type. First, we identify for each defect type, the specific sites in the chosen supercell (see Section 4.5 for details) that result in the highest and lowest formation energy in the neutral charge state (Figure 7a). We assume that, when different charge states of the defect are considered, these two specific sites will allow us to sample the range of formation energies. Next, for each defect type, we calculate the defect formation energy in multiple charge states but only at the two sites identified in the first step. Ultimately, we are able to calculate the range of formation energies as shown in Figure $7 \mathrm{~b}$ and $7 \mathrm{c}$ and assess dopability of alloys. Overall, this adapted approach significantly reduces the computational overhead while providing a reliable assessment of alloy dopability.

Figure 7 a shows the range of formation energy for each defect type in the neutral charge state. The atomic site indices corresponding to the highest and lowest formation energy are labeled in Figure 7a. These sites are labeled in the supercell structure shown in Figure S4. The range of defect formation energies when all plausible charge states are considered are shown in Figures $7 \mathrm{~b}$ and $7 \mathrm{c}$ for the $\mathrm{Ba}_{0.75} \mathrm{Sr}_{1.25} \mathrm{CdP}_{2}$ alloy.

In our convex hull analysis, we find 18 distinct four-phase stability regions of $\mathrm{Ba}_{0.75} \mathrm{Sr}_{1.25} \mathrm{CdP}_{2}$ (Table S9) in the quaternary BaSr-Cd-P phase space. In reality, the alloy composition space i.e., $x$ is continuous, but we perform the convex hull analysis assuming 
(a) statistics of neutral defects

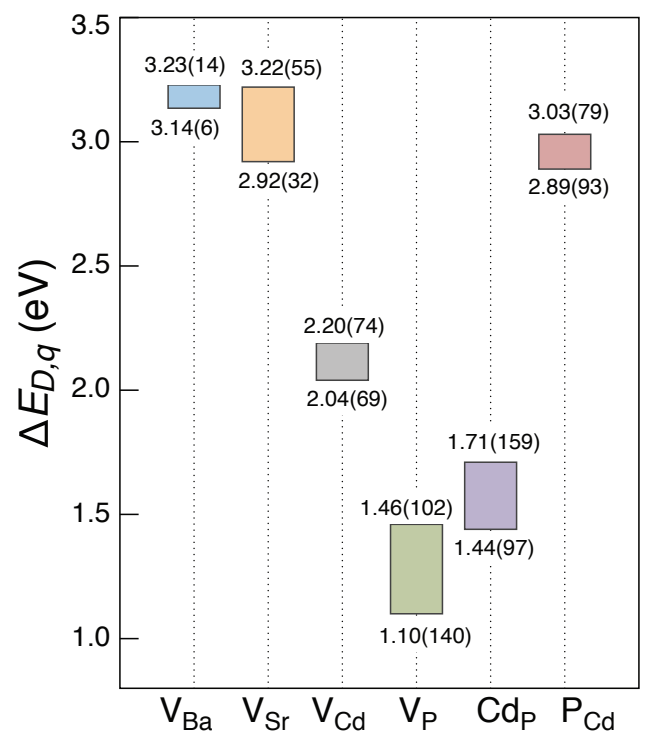

(b) most $n$-type dopable

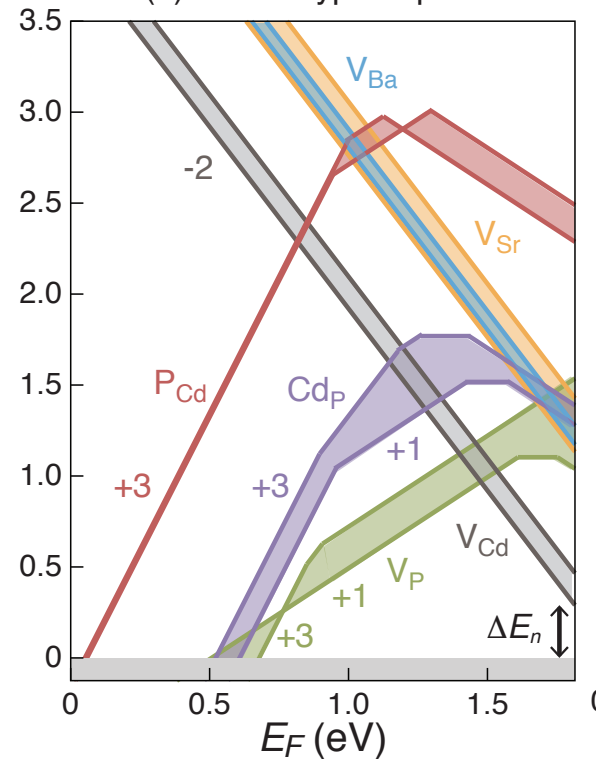

(c) most $p$-type dopable

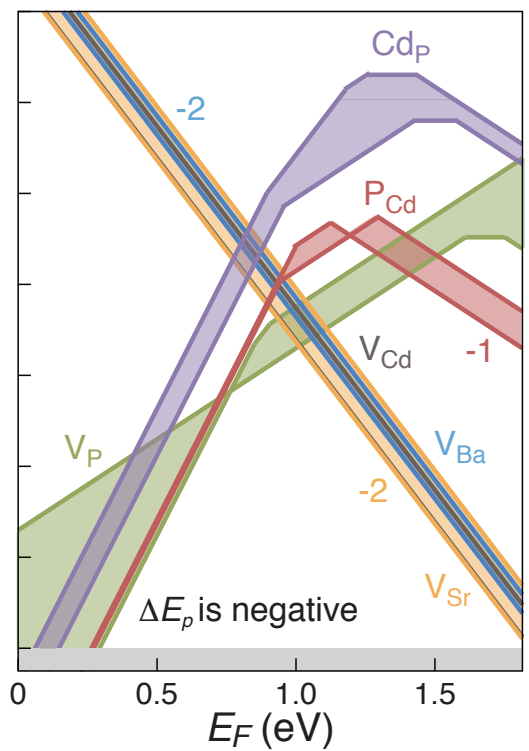

Fig. 7 (a) Range of formation energy (shaded bar) of native point defects $\left(\Delta E_{D, q}\right)$ of $\mathrm{Ba}_{0.75} \mathrm{Sr}_{1.25} \mathrm{CdP}_{2}$ in the neutral charge state. For each defect, the atomic indices corresponding to the maximum and minimum $\Delta E_{D, q}$ are labeled in parentheses, and marked in the crystal structure in Figure $S 4$. (b) Range of formation energy (shaded region) of each native defect in different charge states as a function of the Fermi energy ( $\left.E_{\mathrm{F}}\right)$ for the chemical potential/growth condition that maximizes the $n$-type dopability window $\left(\Delta E_{n}\right) . \Delta E_{n}$ is large and positive suggesting $\mathrm{Ba}_{0.75} \mathrm{Sr}_{1.25} \mathrm{CdP}_{2}$ is $n$-type dopable. (c) Range of formation energy of native defects for the chemical potential condition that maximizes the $p$-type dopability window $\left(\Delta E_{p}\right)$. Since $\Delta E_{p}$ is large and negative, $\mathrm{Ba}_{0.75} \mathrm{Sr}_{1.25} \mathrm{CdP}_{2}$ is not $p$-type dopable.

discrete alloy compositions that are marked in Figure 3. The overall conclusions about the dopability will remain unchanged even if a continuous composition space is considered in the convex hull analysis. In the context of $n$-type dopability, cation vacancies $\left(\mathrm{V}_{\mathrm{Ba}}\right.$, $\mathrm{V}_{\mathrm{Sr}}, \mathrm{V}_{\mathrm{Cd}}$ ) are found to be the "killer" electron-compensating acceptor defects that may limit $n$-type doping. The formation of cation vacancies will be suppressed under cation-rich growth conditions. As such, we identified the four-phase stability region that corresponds to the most cation-rich conditions such that the $n$ type dopability window $\Delta E_{n}$ is maximized.

Figure $7 \mathrm{~b}$ shows the range of formation energy (shaded regions) of each native defect under the most favorable chemical potential conditions that maximizes $\Delta E_{n}$ (P-1 in Table S9, where $\mathrm{Ba}_{0.75} \mathrm{Sr}_{1.25} \mathrm{CdP}_{2}$ is in equilibrium with $\mathrm{SrCd}_{11}, \mathrm{SrCd}_{2}$, $\left.\mathrm{Ba}_{0.60} \mathrm{Sr}_{1.40} \mathrm{CdP}_{2}\right)$. Under these conditions, Cd vacancy $\left(\mathrm{V}_{\mathrm{Cd}}\right)$ is the acceptor defect with lowest formation energy. A positive $\Delta E_{n}$ of $\sim 0.3 \mathrm{eV}$ suggests that $\mathrm{Ba}_{0.75} \mathrm{Sr}_{1.25} \mathrm{CdP}_{2}$ alloy is $n$-type dopable.

In contrast, we find that $\mathrm{Ba}_{0.75} \mathrm{Sr}_{1.25} \mathrm{CdP}_{2}$ is not $p$-type dopable because of the low formation energy of the native donor defects $V_{P}$ and $P_{C d}$ (Figure $7 c$ ). This is atypical for Zintl phases that are commonly $p$-type seminconductors. ${ }^{42}$ Even under the most favorable chemical potential conditions (P-16 in Table S9, where $\mathrm{Ba}_{0.75} \mathrm{Sr}_{1.25} \mathrm{CdP}_{2}$ is in equilibrium with $\operatorname{SrCd}_{2} \mathrm{P}_{2}, \operatorname{SrP}_{3}$, $\mathrm{Ba}_{0.9} \mathrm{Sr}_{1.1} \mathrm{CdP}_{2}$ ), the $p$-type dopability window $\Delta E_{p}$ is large and negative, which means that holes generated by extrinsic dopants will be compensated by the electrons from the native donors. Nevertheless, our focus in this work is to optimize the $n$-type transport of $\mathrm{Ba}_{2(1-x)} \mathrm{Sr}_{2 x} \mathrm{CdP}_{2}$ alloys.

We have previously studied ${ }^{30}$ the native defect energetics of the parent compound, $\mathrm{Ba}_{2} \mathrm{CdP}_{2}$ as well as the ordered $\mathrm{Ba}_{1.0} \mathrm{Sr}_{1.0} \mathrm{CdP}_{2}$. The defect chemistry of $\mathrm{Ba}_{0.75} \mathrm{Sr}_{1.25} \mathrm{CdP}_{2}$ alloy bears a strong qualitative resemblance to that of $\mathrm{Ba}_{2} \mathrm{CdP}_{2}$ and ordered $\mathrm{Ba}_{1.0} \mathrm{Sr}_{1.0} \mathrm{CdP}_{2}$. In both, the ordered compounds and the disordered alloy, we find that the low-energy acceptor defects are the cation vacancies $\left(\mathrm{V}_{\mathrm{Cd}}, \mathrm{V}_{\mathrm{Ba}}, \mathrm{V}_{\mathrm{Sr}}\right)$ with $\mathrm{V}_{\mathrm{Cd}}$ being the lowest among them under most growth conditions. Similarly, $\mathrm{V}_{\mathrm{P}}$ and $\mathrm{P}_{\mathrm{Cd}}$ are the low-energy native donor defects that limit $p$-type doping in the parent compound as well as the alloy. However, there are quantitative differences in the defect chemistry of $\mathrm{Ba}_{2} \mathrm{CdP}_{2}$ and $\mathrm{Ba}_{0.75} \mathrm{Sr}_{1.25} \mathrm{CdP}_{2}$, namely, $\Delta E_{n}$ in $\mathrm{Ba}_{2} \mathrm{CdP}_{2}(\sim 0.7 \mathrm{eV})$ is larger than in $\mathrm{Ba}_{0.75} \mathrm{Sr}_{1.25} \mathrm{CdP}_{2}(\sim 0.4 \mathrm{eV})$.

The decrease in the $n$-type dopability window could be attributed to the increase in the band gap with increase in $\mathrm{Sr}$ - $\mathrm{Ba}_{2} \mathrm{CdP}_{2}$ has a smaller band gap $(1.43 \mathrm{eV})^{30}$ compared to $\mathrm{Ba}_{0.75} \mathrm{Sr}_{1.25} \mathrm{CdP}_{2}(1.81 \mathrm{eV})$. If one were to assume that a fraction of the band gap increase results in a shift of the conduction band minimum to higher energy, then a simple extrapolation of the $\mathrm{V}_{\mathrm{Cd}}$ formation energy (line with negative slope -2 in Figure $7 \mathrm{~b})$ could explain the decrease in $\Delta E_{n}$. Based on this observation, we formulate that the alloy compositions with lower Sr content (smaller $x$ ) are optimal for maximizing $n$-type dopability, as quantified by $\Delta E_{n}$. Taking the electronic and thermal transport, and dopability factors into consideration, we computationally predict that $\mathrm{Ba}_{2(1-x)} \mathrm{Sr}_{2 x} \mathrm{CdP}_{2}$ alloys with $x=0.35-0.70$ are optimal, with lower $x$ in that range preferred for larger dopability $\left(\Delta E_{n}\right)$. 


\section{Conclusions}

In summary, we have developed a computational framework for systematic optimization of the transport properties and dopability of thermoelectric alloys. We simultaneously consider the effects of alloying on electronic and thermal transport, and dopability, which makes this computational framework unique compared to other computational studies of thermoelectric alloys. We apply this framework to identify the optimal compositions of $n$-type $\mathrm{Ba}_{2(1-x)} \mathrm{Sr}_{2 x} \mathrm{CdP}_{2}$ Zintl thermoelectric alloys by achieving conduction band convergence, minimization of alloy scattering of electrons, lowering of $\kappa_{L}$, and ensuring $n$-type dopability. Our computational framework can be used to accelerate the design of thermoelectric alloys with desired transport and doping properties. Such a framework can be used to design alloys for other functional applications beyond thermoelectrics.

We understand that the computational framework presented in this work represents a step towards creating a more comprehensive framework that also takes into account the effects of alloying on phonon scattering, for example, by considering effective phonon band structures ${ }^{43}$ and by developing computationally cheaper models to directly estimate the carrier mobility in alloys, akin to the semi-empirical models developed by one of the co-authors. ${ }^{38}$ Looking forward, data-driven methods will play a key role in computational alloy design. Among the promising developments are the application of machine learning (ML) to design of high-entropy alloys, ${ }^{22-24}$ and the development of new ML algorithms that optimize fractional compositions. ${ }^{44}$ In concert with computational predictions, high-throughput bulk and thin-film synthesis will play a role in validation of the computational predictions. We may envision the synergy of first-principles alloy modeling (demonstrated in this work), data-driven methods, and high-throughput bulk synthesis to tackle the formidable challenge of alloy design and optimization.

\section{Acknowledgements}

JQ is funded by the NSF DIGI-MAT program, grant no. 1922758. $A B$ acknowledges support from the University of Delaware's undergraduate research program. The experimental part of this research, led by SB and conducted at the University of Delaware, received financial support from the US Department of Energy through a grant DE-SC0008885. P.G. acknowledges support from NSF through award DMR-2102409. The research was performed using computational resources sponsored by the Department of Energy's Office of Energy Efficiency and Renewable Energy and located at the NREL. The views expressed in the article do not necessarily represent the views of the DOE or the U.S. Government.

\section{Methods}

\subsection{Structure Relaxation and Bulk Modulus Calculation}

First-principles calculations, including structural relaxations, were performed with density functional theory (DFT) using the Vienna Ab Initio Simulation Package (VASP) software package. ${ }^{45}$ The generalized gradient approximation (GGA) of Perdew-BurkeErnzerhof (PBE) was used as the exchange correlation func- tional. ${ }^{46}$ The core and valence electrons were treated with the projector-augmented wave (PAW) method. ${ }^{47} \mathrm{~A}$ plane-wave energy cutoff of $340 \mathrm{eV}$ was used, and an automatically generated $\Gamma$-centered regular $k$-point mesh was used to sample the Brillouin zone. Bulk modulus $(B)$ was determined by fitting the BirchMurnaghan equation of state to a set of total energies computed at different volumes that were expanded and contracted around the equilibrium volume). ${ }^{48}$

\subsection{SQS Structures and Convergence Tests}

Alloy supercell models were constructed using special quasirandom structures (SQS). ${ }^{27}$ The concept of SQS was developed to mimic random alloys without using very large supercells or using many configurations. The design of SQS involves a stochastic search over many possible configurations of local environments within a given supercell to best reproduce the pairwise correlation of a completely random alloy. An optimal SQS is the one that minimizes the root-mean-square deviation from the random pairwise correlation.

The SQS supercells for each alloy composition were constructed using the Alloy Theoretic Automated Toolkit (ATAT). ${ }^{49}$ In addition to minimizing the deviation from the random pairwise correlation, we also considered different supercell sizes to check for convergence of electronic structure features (band gap, effective masses). The results of the convergence tests for $\mathrm{Ba}_{0.4} \mathrm{Sr}_{1.6} \mathrm{CdP}_{2}$ are summarized in Table S7. We found that a 100 atom SQS supercell with a cutoff distance of $6 \AA$ for pairs and triplets provides sufficiently converged electronic structure properties. We used a 100-atom SQS for all compositions $(x)$ to calculate their free energy of mixing (Figure 3) and effective band structures (Figure 5).

The cations (Ba, Sr) can occupy two distinct Wyckoff positions in the $\mathrm{Ba}_{2(1-x)} \mathrm{Sr}_{2 x} \mathrm{CdP}_{2}$ structure (sites 1 and 2 in Figure 2). To model the site selectivity of the cation disorder, for each composition, we considered different SQS supercells with the disorder constrained to a specific Wyckoff site. Given the equal stoichiometric ratio of sites 1 and 2, for the 50-50 alloy, only one SQS supercell with disorder on both sites was considered. Each SQS supercell was fully relaxed (volume, cell shape, and atomic positions) with DFT using the functionals and parameters described in Section 4.1.

\subsection{Calculation of Free Energy of Mixing}

The free energy of mixing $\Delta G_{\text {mix }}$ in Figure 3 was calculated as $\Delta G_{\text {mix }}=\Delta H_{\text {mix }}-T \Delta S_{\text {mix }}$, where $\Delta H_{\text {mix }}$ and $\Delta S_{\text {mix }}$ are the enthalpy and entropy of mixing, respectively. $\Delta H_{\text {mix }}$ is calculated as,

$$
\Delta H_{\text {mix }}=H_{\text {alloy }}-\sum_{i} w_{i} H_{i}
$$

where $w_{i}$ is the fraction of each parent compound in the alloy. $H_{\text {alloy }}$ is calculated from the DFT total energy of the SQS supercell and $H_{i}$ from the total energy of the unit cell of the parent compounds. We assume that the configurational entropy due to disorder in the alloy is the primary contribution to $\Delta S_{\text {mix }}$. The configurational entropy is calculated using the Boltzmann's for- 
mula as,

$$
\Delta S_{\text {config }}=\sum_{j} y_{j} \ln \left(y_{j}\right)
$$

where $j$ is the chemical species with fractional occupation, and $y_{j}$ is the fraction of the disordered sites occupied by $j$. The fractions $y_{j}$ should add up to 1 i.e., $\sum_{j} y_{j}=1$. The vibrational entropy change is assumed to be zero due to the approximate cancellation of the vibrational entropy between the alloy and the parent compounds. A typical synthesis temperature of $823 \mathrm{~K}$ is used to compute $\Delta G_{\text {mix }}$ in Figure 3. The trends remain unchanged at higher synthesis temperatures.

\subsection{Effective Band Structures}

We use the effective band structure (EBS) ${ }^{28}$ to interpret the conduction band electronic structure of the alloys. The EBS is obtained by unfolding the SQS supercell bands to the primitive cell Brillouin zone using spectral decomposition. Each eigenstate in the primitive cell is assigned a spectral weight that represents how well the Bloch character of that eigenstate has been preserved when unfolded from the supercell wavefunctions. The spectral weight is defined by:

$$
P_{m \vec{K}}(\vec{k})=\sum_{n}\left|\left\langle\psi_{m \vec{K}}^{S C} \mid \psi_{n \vec{k}}^{P C}\right\rangle\right|^{2}
$$

where $\left|\psi_{m \vec{K}}^{S C}\right\rangle$ are the eigenstates in the supercell (SC) at wave vector $\vec{K}$ and band index $m$. Similarly, $\left|\psi_{n \vec{k}}^{P C}\right\rangle$ are the eigenstates in the primitive cell (PC) at wave vector $\vec{k}$ and band index $n$. The spectral function is then defined as,

$$
A(\vec{k}, E)=\sum_{m} P_{m \vec{K}}(\vec{k}) \delta\left(E-E_{m}(\vec{K})\right)
$$

where $\delta$ is the Delta function. Finally, the EBS is obtained by integrating the spectral function $(A(\vec{k}, E))$ in an infinitesimal energy window $d E$ :

$$
\delta N\left(\vec{k}_{i}, E_{j}\right)=\int_{E_{j}-\delta E / 2}^{E_{j}+\delta E / 2} A\left(\vec{k}_{i}, E\right) d E
$$

where $\delta N\left(\vec{k}_{i}, E_{j}\right)$ gives the number of SC bands crossing PC wave vector $k_{i}$ and energy level $E_{j}$, which is shown as the scale bar in Figure 5. EBS is calculated along the special $k$-point paths in the Brillouin zone of $\mathrm{Ba}_{2} \mathrm{CdP}_{2}$. We use 40 interpolation points between each pair of high-symmetry $k$ points of the PC. We also calculate the EBS with 20 interpolation points (see comparison in Figure S3) to confirm that there are no interpolation artifacts. The EBSs are calculated using a modified version of the BandUP code. ${ }^{50}$

\subsection{Defect Energetics}

We adapted the standard supercell approach ${ }^{29}$ to calculate the defect energetics. First, we build a 40-atom SQS structure as the "unit cell" for the $\mathrm{Ba}_{0.75} \mathrm{Sr}_{1.25} \mathrm{CdP}_{2}(x=0.625)$ alloy. Next, we create a $2 \times 2 \times 1$ supercell of the 40 -atom SQS such that the supercell contains 160 atoms. We perform convergence test with 120 -atom SQS to show that the 40-atom SQS reproduces the EBS and related electronic properties of the larger SQS supercell (Table S8). The defect formation energy $\left(\Delta E_{D, q}\right)$ is calculated from DFT total energies as follows:

$$
\Delta E_{D, q}=E_{D, q}-E_{\mathrm{host}}+\sum_{i} n_{i} \mu_{i}+q E_{\mathrm{F}}+E_{\mathrm{corr}}
$$

where $\Delta E_{\mathrm{D}, q}$ is the formation energy of a defect $D$ in charge state $q, E_{D, q}$ is the total energy of the supercell containing defect $D$ in charge state $q$, and $E_{\text {host }}$ is the total energy of the defect-free, neutral host supercell. $E_{\mathrm{F}}$ is the Fermi energy, which referenced to the valence band maximum (VBM) i.e. $E_{\mathrm{F}}=0$ at the VBM. $E_{\mathrm{corr}}$ term contains the finite-size corrections, which are calculated following the methodology of Lany and Zunger ${ }^{29}$. The finite-size corrections comprises: (1) image charge correction for charged defects, (2) potential alignment correction for charged defects, and (3) band-filling correction for shallow defects. The pyladadefects software ${ }^{51}$ is used to generate the defect supercells and to calculate the finite-size corrections.

The chemical potential of elemental species $i$ is denoted by $\mu_{i}$ and $n_{i}$ is the number of atoms of species $i$ added $\left(n_{i}<0\right)$ or removed $\left(n_{i}>0\right)$ from the host supercell to form the defect $D$. $\mu_{i}$ is expressed as $\mu_{i}=\mu_{0}+\Delta \mu$, where $\mu_{0}$ is the reference elemental chemical potential and $\Delta \mu_{i}$ is the deviation from the reference value. $\mu_{0}$ are fitted to a set of experimentally-measured formation enthalpy of compounds in the quaternary Ba-Sr-Cd-P chemical space. The fitted $\mu_{0}$ values are listed in Table S10. For a material, the accessible range of $\Delta \mu_{i}$ is constrained by the condition of its thermodynamic phase stability and is calculated through a convex hull analysis. Table S9 lists the $\Delta \mu_{i}$ values at the corners of the polyhedra that defines the region of phase stability of $\mathrm{Ba}_{0.75} \mathrm{Sr}_{1.25} \mathrm{CdP}_{2}$.

The positions of the ions in the defect supercells are relaxed with DFT using a plane-wave energy cutoff of $340 \mathrm{eV}$. The Brillouin zone of the supercell is sampled with a $\Gamma$-centered $2 \times 2 \times 2$ $k$-point grid. We consider native vacancies $\left(\mathrm{V}_{\mathrm{Ba}}, \mathrm{V}_{\mathrm{Sr}}, \mathrm{V}_{\mathrm{Cd}}, \mathrm{V}_{\mathrm{P}}\right)$ and anti-site defects $\left(\mathrm{Cd}_{\mathrm{P}}, \mathrm{P}_{\mathrm{Cd}}\right)$. The interstitial defects $\mathrm{Ba}_{\mathrm{i}}$ and $\mathrm{Sr}_{\mathrm{i}}$ are not considered in this work because they are expected to have high formation energy due to their large ionic radii. For each defect type, we consider all unique Wyckoff positions in the structure and multiple charge states $q=-3,-2,-1,0,1,2,3$. The underestimation of the band gap in DFT is rectified by applying individual valence and conduction band edge shifts (relative to the DFT-computed band edges) as determined from GW quasiparticle energies. ${ }^{29,52}$ Because of the direct band gap at $\Gamma$, the GW quasi-particle energies are calculated for the 40-atom SQS using a $\Gamma$-point only $k$-point grid.

\subsection{Material Synthesis and Characterization}

All manipulations and procedures were performed either in an inert argon environment or under a vacuum. The elements used in the synthesis of the title compounds ( $\mathrm{Ba}, \mathrm{Cd}, \mathrm{P}, \mathrm{Pb}$, and $\mathrm{Sr}$ ) were sourced from either Sigma-Aldrich or Alfa Aesar and carry a stated purity of, at least, $99 \mathrm{wt} \%$. Due to the high purity of the elements, they were used as received. The elements were loaded, in desired stoichiometric ratios, into alumina crucibles, 
which were subsequently flame sealed in silica jackets. The vessels were then placed into a programmable muffle furnace for heat treatment. The compounds synthesized, as a result of this inquiry into the Ba-Sr-Cd-P phase diagram, were made using a standard $\mathrm{Pb}$ flux technique wherein the elements were loaded with an excess of $\mathrm{Pb}$ intended as a solvent for the high temperature reaction. The heat treatment closely followed the optimized synthetic procedures used to obtain the parent $\mathrm{Yb}_{2} \mathrm{CdSb}_{2}$ structure: 1 the elements were rapidly heated to a peak temperature of $1233 \mathrm{~K}$, allowed to equilibrate at this temperature for $24 \mathrm{hrs}$, and then slowly cooled to $773 \mathrm{~K}$ at a rate of $5 \mathrm{~K} / \mathrm{hr}$. Once the temperature of $773 \mathrm{~K}$ was reached, the vessels were removed from the furnace and centrifuged at high speeds to remove the excess $\mathrm{Pb}$ flux. Due to the experience with air sensitivity of compounds, the vessel was then transferred into an argon-filled glovebox for further manipulations. The crystals presented in this work were extracted from the matrix as black, needle-like crystals that and were used for further structural studies.

\subsection{Single Crystal X-ray Diffraction}

Single crystal X-ray diffraction data were collected for all of the compounds using the following procedure: Crystals, of suitable quality, were identified, while immersed in Paratone-N oil, under a high-powered microscope and then subsequently cut to size. The freshly cut crystals were then scooped up by a MiTeGen plastic loop and placed into the goniometer of a Bruker ApexII diffractometer, which utilizes a sealed source Mo K $\alpha$ radiation $(0.71073 \AA$ ) for diffraction. A constant temperature of $200 \mathrm{~K}$ was maintained by cold nitrogen vapor that was obtained from boiling liquid $\mathrm{N}_{2}$. This cold nitrogen stream also served to preserve an inert environment by hardening the Paratone-N oil, forming a hard shell around the crystal such that the decomposition of the material was prevented.

Data were collected, subsequently integrated, and corrected for absorption using the Bruker-supplied software ${ }^{53}$. The structures were solved with ShelXT ${ }^{54}$, using Olex2 ${ }^{55}$ as the graphical interface. Further refinement of the crystal structures was performed using SHELXL, ${ }^{56}$ which utilizes a full-matrix least-squares method, on $F^{2}$. Such refinements include the necessary modeling of occupational disorder at the divalent alkaline earth metal sites, such that the measured electron density at each site is properly accounted for. The important crystallographic data from the series of structures with the general formula, $\mathrm{Ba}_{2(1-x)} \mathrm{Sr}_{2 x} \mathrm{CdP}_{2}$, can be found in Table $\mathrm{S} 1$ to $\mathrm{S} 6$ for the Ba-poor and Ba-rich compositions respectively.

\section{References}

1 M. A. Der Maur, K. Lorenz and A. Di Carlo, Opt. Quantum Electron, 2012, 44, 83.

2 W. K. Metzger, S. Grover, D. Lu, E. Colegrove, J. Moseley, C. L. Perkins, X. Li, R. Mallick, W. Zhang, R. Malik et al., Nat. Energy, 2019, 4, 837.

3 M. Hu, M. Chen, P. Guo, H. Zhou, J. Deng, Y. Yao, Y. Jiang, J. Gong, Z. Dai, Y. Zhou et al., Nat. Commun, 2020, 11, 1.
4 T. Bernges, S. P. Culver, N. Minafra, R. Koerver and W. G. Zeier, Inorg. Chem., 2018, 57, 13920.

5 M. Akiyama, T. Kamohara, K. Kano, A. Teshigahara, Y. Takeuchi and N. Kawahara, Adv. Mater., 2009, 21, 593.

6 Z. Tian, J. Garg, K. Esfarjani, T. Shiga, J. Shiomi and G. Chen, Phys. Rev. B, 2012, 85, 184303.

7 A. Ludwig, npj Comput. Mater., 2019, 5, 1.

8 L.-D. Zhao, H. Wu, S. Hao, C.-I. Wu, X. Zhou, K. Biswas, J. He, T. P. Hogan, C. Uher, C. Wolverton et al., Energy Environ. Sci., 2013, 6, 3346.

9 K. P. Devlin, S. Chen, D. Donadio and S. M. Kauzlarich, Inorg. Chem., 2021, 60, 13596.

10 T. J. Slade, K. Pal, J. A. Grovogui, T. P. Bailey, J. Male, J. F. Khoury, X. Zhou, D. Y. Chung, G. J. Snyder, C. Uher et al., J. Am. Chem. Soc., 2020, 142, 12524.

11 K. Imasato, S. D. Kang, S. Ohno and G. J. Snyder, Mater. Horiz., 2018, 5, 59.

12 W. Liu, X. Tan, K. Yin, H. Liu, X. Tang, J. Shi, Q. Zhang and C. Uher, Phys. Rev. Lett., 2012, 108, 166601.

13 S. Ohno, K. Imasato, S. Anand, H. Tamaki, S. D. Kang, P. Gorai, H. K. Sato, E. S. Toberer, T. Kanno and G. J. Snyder, Joule, 2018, 2, 141.

14 B. R. Ortiz, J. M. Adamczyk, K. Gordiz, T. Braden and E. S. Toberer, Mol. Syst. Des. Eng, 2019, 4, 407.

15 M. Y. Tafti, S. Ballikaya, A. M. Khachatourian, M. Noroozi, M. Saleemi, L. Han, N. V. Nong, T. Bailey, C. Uher and M. S. Toprak, RSC Adv., 2016, 6, 111457.

16 F. Ricci, A. Dunn, A. Jain, G.-M. Rignanese and G. Hautier, J. Mater. Chem. A, 2020, 8, 17579.

17 J. Qu, V. Stevanović, E. Ertekin and P. Gorai, J. Mater. Chem. A, 2020, 8, 25306.

18 H. Zhu, G. Hautier, U. Aydemir, Z. M. Gibbs, G. Li, S. Bajaj, J.H. Pöhls, D. Broberg, W. Chen, A. Jain et al., J. Mater. Chem. C, 2015, 3, 10554.

19 A. Faghaninia, G. Yu, U. Aydemir, M. Wood, W. Chen, G.-M. Rignanese, G. J. Snyder, G. Hautier and A. Jain, Phys. Chem. Chem. Phys, 2017, 19, 6743.

20 J. M. Adamczyk, L. C. Gomes, J. Qu, G. A. Rome, S. M. Baumann, E. Ertekin and E. S. Toberer, Chem. Mater, 2020, 33, 359.

21 S. Barabash, V. Ozolins and C. Wolverton, Phys. Rev. Lett., 2008, 101, 155704.

22 R. Feng, M. C. Gao, C. Lee, M. Mathes, T. Zuo, S. Chen, J. A. Hawk, Y. Zhang and P. K. Liaw, Entropy, 2016, 18, 333.

23 C. Wen, Y. Zhang, C. Wang, D. Xue, Y. Bai, S. Antonov, L. Dai, T. Lookman and Y. Su, Acta Mater., 2019, 170, 109.

24 Z. Zhou, Y. Zhou, Q. He, Z. Ding, F. Li and Y. Yang, npj Comput. Mater., 2019, 5, 1-9.

25 B. S. Shariat, Q. Meng, A. S. Mahmud, Z. Wu, R. Bakhtiari, J. Zhang, F. Motazedian, H. Yang, G. Rio, T.-h. Nam et al., Mater. Des., 2017, 124, 225.

26 Y. Li, Z. Feng, L. Hao, L. Huang, C. Xin, Y. Wang, E. Bilotti, K. Essa, H. Zhang, Z. Li et al., Adv. Mater. Technol., 2020, 5, 1900981. 
27 A. Zunger, S.-H. Wei, L. Ferreira and J. E. Bernard, Phys. Rev. Lett., 1990, 65, 353.

28 V. Popescu and A. Zunger, Phys. Rev. Lett., 2010, 104, 236403.

29 S. Lany and A. Zunger, Phys. Rev. B, 2008, 78, 235104.

30 A. Balvanz, J. Qu, S. Baranets, E. Ertekin, P. Gorai and S. Bobev, Chem. Mater., 2020, 32, 10697.

31 P. Gorai, H. Long, E. Jones, S. Santhanagopalan and V. Stevanović, J. Mater. Chem. A, 2020, 8, 3851.

32 B. Saparov, M. Saito and S. Bobev, J. Solid State Chem., 2011, 184, 432.

33 J. Wang, M. Yang, M.-Y. Pan, S.-Q. Xia, X.-T. Tao, H. He, G. Darone and S. Bobev, Inorg. Chem., 2011, 50, 8020.

34 S.-q. Xia and S. Bobev, J. Am. Chem. Soc., 2007, 129, 4049.

35 G. Tan, S. Hao, R. C. Hanus, X. Zhang, S. Anand, T. P. Bailey, A. J. E. Rettie, X. Su, C. Uher, V. P. Dravid, G. J. Snyder, C. Wolverton and M. G. Kanatzidis, ACS Energy Lett., 2018, 3, 705.

36 R. Hanus, M. T. Agne, A. J. E. Rettie, Z. Chen, G. Tan, D. Y. Chung, M. G. Kanatzidis, Y. Pei, P. W. Voorhees and G. J. Snyder, Adv. Mater., 2019, 31, 1900108.

37 A. Togo, L. Chaput and I. Tanaka, Phys. Rev. B, 2015, 91, 094306.

38 J. Yan, P. Gorai, B. Ortiz, S. Miller, S. A. Barnett, T. Mason, V. Stevanović and E. S. Toberer, Energy Environ. Sci., 2015, 8, 983.

39 P. Gorai, V. Stevanovic and E. S. Toberer, Nat. Rev. Mater., 2017, 2, 1.

40 M. Y. Toriyama, J. Qu, G. J. Snyder and P. Gorai, J. Mater. Chem. A, 2021.
41 C. Freysoldt, B. Grabowski, T. Hickel, J. Neugebauer, G. Kresse, A. Janotti and C. G. Van de Walle, Rev. Mod. Phys, 2014, 86, 253.

42 P. Gorai, A. Goyal, E. S. Toberer and V. Stevanović, J. Mater. Chem. A, 2019, 7, 19385.

43 J. M. Skelton, Lattice dynamics of Pnma $\operatorname{Sn}\left(S_{1-x} S_{x}\right)$ solid solutions: energetics, phonon spectra and thermal transport, 2020.

44 A. Y.-T. Wang, S. K. Kauwe, R. J. Murdock and T. D. Sparks, npj Comput. Mater., 2021, 7, 1.

45 G. Kresse and J. Furthmüller, Phys. Rev. B, 1996, 54, 11169.

46 J. P. Perdew, K. Burke and M. Ernzerhof, Phys. Rev. Lett., 1996, 77, 3865.

47 P. E. Blöchl, Phys. Rev. B, 1994, 50, 17953.

48 F. Birch, J. Geophys. Res., 1952, 57, 227.

49 A. van de Walle, Calphad, 2009, 33, 266.

50 P. V. C. Medeiros, S. Stafström and J. Björk, Phys. Rev. B, 2014, 89, 041407.

51 A. Goyal, P. Gorai, H. Peng, S. Lany and V. Stevanovic, Comput. Mater. Sci., 2017, 130, 1.

52 H. Peng, D. O. Scanlon, V. Stevanovic, J. Vidal, G. W. Watson and S. Lany, Phys. Rev. B, 2013, 88, 115201.

53 G. Sheldrick, University of Göttingen, Göttingen, Germany, 2014.

54 G. Sheldrick and A. C. Shelxs97, Acta Crystallogr., 2015, 71, 3.

55 O. V. Dolomanov, L. J. Bourhis and R. J. Gildea, J. Appl. Crystallogr., 2009, 42, 339.

56 G. M. Sheldrick, Acta Crystallogr., 2015, 71, 3. 


\title{
- Electronic Supplementary Information -
}

\section{Computational Design of Thermoelectric Alloys Through Optimization of Transport and Dopability}

\author{
Jiaxing Qu, ${ }^{\dagger}$ Adam Balvanz, ${ }^{\ddagger}$ Sviatoslav Baranets, ${ }^{\ddagger}$ Svilen Bobev, ${ }^{\ddagger}$ and Prashun Gorai*, \\ $\dagger$ University of Illinois at Urbana-Champaign, Urbana, IL 61801 \\ $\ddagger$ University of Delaware, Newark, DE 19716 \\ ฯ Colorado School of Mines, Golden, CO 80401 \\ E-mail: pgorai@mines.edu
}




\section{Atomic Coordinates and Equivalent Displacement Parameters}

Table S1: Fractional atomic coordinates and equivalent displacement parameters $\left(\AA^{2}\right)$ for (1) $\mathrm{Sr}_{2} \mathrm{CdP}_{2}^{*}$ and (2) the Ba-poor compositions of the solid solution $\mathrm{Ba}_{2(1-x)} \mathrm{Sr}_{2 x} \mathrm{CdP}_{2}$.

${ }^{*} \mathrm{Sr}_{2} \mathrm{CdP}_{2}$ was not resynthesized in this study, but is presented for reference. For information on $\mathrm{Sr}_{2} \mathrm{CdP}_{2}$, please see our previous work. ${ }^{1 \dagger} U_{\text {eq }}$ is defined as one third of the trace of the orthogonalized $U_{i j}$ tensor.

\begin{tabular}{|c|c|c|c|c|}
\hline Composition & $x$ & $y$ & $z$ & $U_{\mathrm{eq}}^{\dagger}$ \\
\hline \multicolumn{5}{|l|}{$\mathbf{S r}_{2} \mathbf{C d P}_{2}$} \\
\hline Sr1 & 0 & $0.29673(9)$ & $0.5970(2)$ & $0.0143(4)$ \\
\hline $\mathrm{Sr} 2$ & 0 & $0.46350(1)$ & $0.2560(2)$ & $0.0129(4)$ \\
\hline $\mathrm{Cd}$ & 0 & $0.09625(7)$ & $0.4103(2)$ & $0.0136(3)$ \\
\hline $\mathrm{P} 1$ & 0 & $0.06150(3)$ & $0.0518(6)$ & $0.0116(9)$ \\
\hline $\mathrm{P} 2$ & 0 & $0.32570(3)$ & $0.0000(7)$ & $0.0130(1)$ \\
\hline \multicolumn{5}{|c|}{$\mathbf{B a}_{0.4} \mathbf{S r}_{1.6} \mathbf{C d P}_{2}$} \\
\hline $\mathrm{Ba} 1 / \mathrm{Sr} 1$ & 0 & $0.29689(4)$ & $0.5944(1)$ & 0.0150 \\
\hline $\mathrm{Sr} 2$ & 0 & $0.46517(6)$ & $0.2601(1)$ & $0.0125(2)$ \\
\hline $\mathrm{Cd}$ & 0 & $0.09531(4)$ & $0.41551(9)$ & $0.0138(2)$ \\
\hline $\mathrm{P} 1$ & 0 & $0.0598(2)$ & $0.0549(4)$ & $0.0116(6)$ \\
\hline P2 & 0 & $0.3283(2)$ & $0.0058(4)$ & $0.0137(6)$ \\
\hline \multicolumn{5}{|c|}{$\mathbf{B a}_{0.6} \mathbf{S r}_{1.4} \mathbf{C d P}_{2}$} \\
\hline $\mathrm{Ba} 1 / \mathrm{Sr} 1$ & 0 & $0.29707(4)$ & $0.5927(1)$ & $0.0137(3)$ \\
\hline $\mathrm{Sr} 2$ & 0 & $0.46568(7)$ & $0.2608(2)$ & $0.0112(2)$ \\
\hline $\mathrm{Cd}$ & 0 & $0.09491(5)$ & $0.41614(9)$ & $0.0126(2)$ \\
\hline $\mathrm{P} 1$ & 0 & $0.0593(2)$ & $0.0556(4)$ & $0.0109(6)$ \\
\hline P2 & 0 & $0.3294(2)$ & $0.0066(4)$ & $0.0139(7)$ \\
\hline \multicolumn{5}{|c|}{$\mathbf{B a}_{0.7} \mathbf{S r}_{1.3} \mathbf{C d P} \mathbf{P}_{2}$} \\
\hline $\mathrm{Ba1} / \mathrm{Sr} 1$ & 0 & $0.29717(3)$ & $0.5903(1)$ & $0.0112(2)$ \\
\hline $\mathrm{Sr} 2$ & 0 & $0.46632(5)$ & $0.2607(2)$ & $0.0092(2)$ \\
\hline $\mathrm{Cd}$ & 0 & $0.09454(4)$ & $0.41671(9)$ & $0.0107(2)$ \\
\hline P1 & 0 & $0.0585(2)$ & 0.0556 & 0.0089 \\
\hline $\mathrm{P} 2$ & 0 & $0.3304(2)$ & $0.0074(4)$ & $0.0115(6)$ \\
\hline \multicolumn{5}{|c|}{$\mathbf{B a}_{0.9} \mathbf{S r}_{1.1} \mathbf{C d P}_{2}$} \\
\hline Ba1/Sr1 & 0 & $0.29721(5)$ & $0.5891(1)$ & 0.0110 \\
\hline $\mathrm{Ba} 2 / \mathrm{Sr} 2$ & 0 & $0.46658(8)$ & $0.2619(2)$ & $0.0093(4)$ \\
\hline $\mathrm{Cd}$ & 0 & $0.09403(5)$ & $0.4180(1)$ & $0.0102(3)$ \\
\hline $\mathrm{P} 1$ & 0 & $0.0577(2)$ & $0.0563(5)$ & $0.0096(8)$ \\
\hline P2 & 0 & $0.3318(2)$ & $0.0084(5)$ & $0.0115(9)$ \\
\hline
\end{tabular}


Table S2: Fractional atomic coordinates and equivalent displacement parameters $\left(\AA^{2}\right)$ for $(1) \mathrm{Ba}_{2} \mathrm{CdP}_{2}^{*}$ and (2) the Ba-rich compositions of the solid solution $\mathrm{Ba}_{2(1-x)} \mathrm{Sr}_{2 x} \mathrm{CdP}_{2}$.

${ }^{*} \mathrm{Ba}_{2} \mathrm{CdP}_{2}$ was not resynthesized in this study, but is presented for reference. For information on $\mathrm{Ba}_{2} \mathrm{CdP}_{2}$, please see our previous work. ${ }^{1}{ }^{\dagger} U_{\text {eq }}$ is defined as one third of the trace of the orthogonalized $U_{i j}$ tensor.

\begin{tabular}{lllll}
\hline Composition & $x$ & $y$ & $z$ & $U_{\text {eq }}^{\dagger}$ \\
\hline
\end{tabular}

$\mathrm{Ba}_{1.1} \mathrm{Sr}_{0.9} \mathbf{C d P}_{2}$

$\begin{array}{llccc}\mathrm{Ba} 1 & 0 & 0.29727(3) & 0.5897(1) & 0.0122(2) \\ \mathrm{Ba} 2 / \mathrm{Sr} 2 & 0 & 0.46636(5) & 0.2624(1) & 0.0100(3) \\ \mathrm{Cd} & 0 & 0.09407(4) & 0.41837(9) & 0.0100(2) \\ \mathrm{P} 1 & 0 & 0.0579(1) & 0.0573(4) & 0.0091(5) \\ \mathrm{P} 2 & 0 & 0.3316(2) & 0.0084(4) & 0.0100(6)\end{array}$

$\mathrm{Ba}_{1.2} \mathbf{S r}_{0.8} \mathbf{C d P}_{2}$

$\mathrm{Ba} 1$

$\mathrm{Ba} 2 / \mathrm{Sr} 2$

$\mathrm{Cd}$

P1

P2

$\mathbf{B a}_{1.3} \mathbf{S r}_{0.7} \mathbf{C d P}_{2}$

Ba1

$\mathrm{Ba} 2 / \mathrm{Sr} 2$

$\mathrm{Cd}$

P1

P2

$\mathbf{B a}_{1.6} \mathbf{S r}_{0.4} \mathbf{C d P}_{2}$

Ba1

$\mathrm{Ba} 2 / \mathrm{Sr} 2$

$\mathrm{Cd}$

P1

P2

\section{$\mathrm{Ba}_{2} \mathbf{C d P}_{2}$}

$\mathrm{Ba} 1$
$\mathrm{Ba} 2$
$\mathrm{Cd}$
$\mathrm{P} 1$
$\mathrm{P} 2$

$$
\begin{gathered}
0.29723(5) \\
0.46540(7) \\
0.09397(6) \\
0.0582(2) \\
0.3315(2)
\end{gathered}
$$

$0.5907(2)$
$0.2629(2)$
$0.4173(1)$
$0.0585(6)$
$0.0058(6)$

$0.0148(2)$

$0.0121(4)$

$0.0127(3)$

$0.0123(8)$

0.016 (1)
0.5925 (1)

0.2626 (1)

$0.4162(1)$

0.0598 (4)

$0.0035(4)$
$0.0137(2)$

$0.0110(3)$

$0.0113(2)$

$0.0103(6)$

$0.0126(7)$

$\begin{array}{cc}0.59502(9) & 0.0130(1) \\ 0.26294(9) & 0.0102(2) \\ 0.41371(8) & 0.0114(2) \\ 0.0620(3) & 0.0100(5) \\ -0.0030(3) & 0.0127(6)\end{array}$

$0.29650(3)$

$0.46206(4)$

0.09428 (4)

0.0581 (1)

$0.3304(2)$

$0.0136(2)$

$0.0128(2)$

$0.0140(3)$

$0.0124(9)$

0.012 (1) 


\section{Selected Bond Distances for $\mathrm{Ba}_{2(1-x)} \mathrm{Sr}_{2 x} \mathrm{CdP}_{2}$}
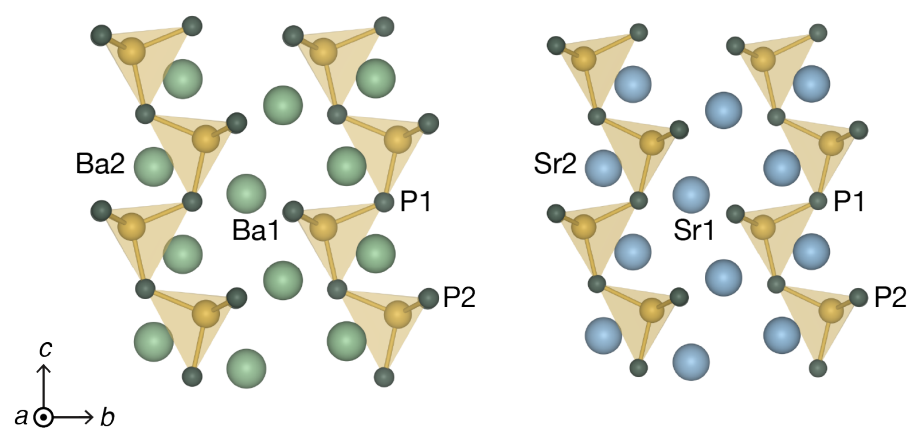

Figure S1: Crystal structures of $\mathrm{Ba}_{2} \mathrm{CdP}_{2}$ and $\mathrm{Sr}_{2} \mathrm{CdP}_{2}$ with labels for different Wyckoff sites of $\mathrm{Ba}, \mathrm{Sr}$, and $\mathrm{P}$.

Table S3: Selected bond distances $(\AA)$.

\begin{tabular}{lll}
\hline Composition & Ba1/Sr1-P1 Distance $(\AA)$ & Ba1/Sr1-P2 Distance $(\AA)$ \\
\hline & & \\
$\mathrm{Ba}_{0.4} \mathrm{Sr}_{1.6} \mathrm{CdP}_{2}$ & $3.264(3) \times 2$ & $3.104(3) \times 2$ \\
$\mathrm{Ba}_{0.6} \mathrm{Sr}_{1.4} \mathrm{CdP}_{2}$ & $3.284(3) \times 2$ & $3.126(3) \times 2$ \\
$\mathrm{Ba}_{0.7} \mathrm{Sr}_{1.3} \mathrm{CdP}_{2}$ & $3.303(2) \times 2$ & $3.148(2) \times 2$ \\
$\mathrm{Ba}_{0.9} \mathrm{Sr}_{1.1} \mathrm{CdP}_{2}$ & $3.332(3) \times 2$ & $3.180(3) \times 2$ \\
$\mathrm{Ba}_{1.1} \mathrm{Sr}_{0.9} \mathrm{CdP}_{2}$ & $3.331(2) \times 2$ & $3.182(2) \times 2$ \\
$\mathrm{Ba}_{1.2} \mathrm{Sr}_{0.8} \mathrm{CdP}_{2}$ & $3.329(3) \times 2$ & $3.188(3) \times 2$ \\
$\mathrm{Ba}_{1.3} \mathrm{Sr}_{0.7} \mathrm{CdP}_{2}$ & $3.338(2) \times 2$ & $3.181(2) \times 2$ \\
$\mathrm{Ba}_{1.6} \mathrm{Sr}_{0.4} \mathrm{CdP}_{2}$ & $3.3416(18) \times 2$ & $3.1943(19) \times 2$ \\
& & \\
\hline
\end{tabular}

3. Selected Bond Angles for $\mathrm{Ba}_{2(1-x)} \mathrm{Sr}_{2 x} \mathrm{CdP}_{2}$

Table S4: Selected bond angles.

\begin{tabular}{lll}
\hline Composition & P1-Cd1-P1 angle $\left(^{\circ}\right)$ & Cd-P1-Cd angle $\left(^{\circ}\right)$ \\
\hline $\mathrm{Ba}_{0.4} \mathrm{Sr}_{1.6} \mathrm{CdP}_{2}$ & $99.21(7)$ & $124.24(9)$ \\
$\mathrm{Ba}_{0.6} \mathrm{Sr}_{1.4} \mathrm{CdP}_{2}$ & $99.03(8)$ & $124.36(12)$ \\
$\mathrm{Ba}_{0.7} \mathrm{Sr}_{1.3} \mathrm{CdP}_{2}$ & $98.81(5)$ & $124.48(10)$ \\
$\mathrm{Ba}_{0.9} \mathrm{Sr}_{1.1} \mathrm{CdP}_{2}$ & $98.68(8)$ & $124.65(13)$ \\
$\mathrm{Ba}_{1.1} \mathrm{Sr}_{0.9} \mathrm{CdP}_{2}$ & $98.80(5)$ & $124.67(10)$ \\
$\mathrm{Ba}_{1.2} \mathrm{Sr}_{0.8} \mathrm{CdP}_{2}$ & $99.38(8)$ & $124.96(15)$ \\
$\mathrm{Ba}_{1.3} \mathrm{Sr}_{0.7} \mathrm{CdP}_{2}$ & $99.79(6)$ & $125.71(11)$ \\
$\mathrm{Ba}_{1.6} \mathrm{Sr}_{0.4} \mathrm{CdP}_{2}$ & $100.95(5)$ & $126.59(9)$ \\
& & \\
\hline
\end{tabular}




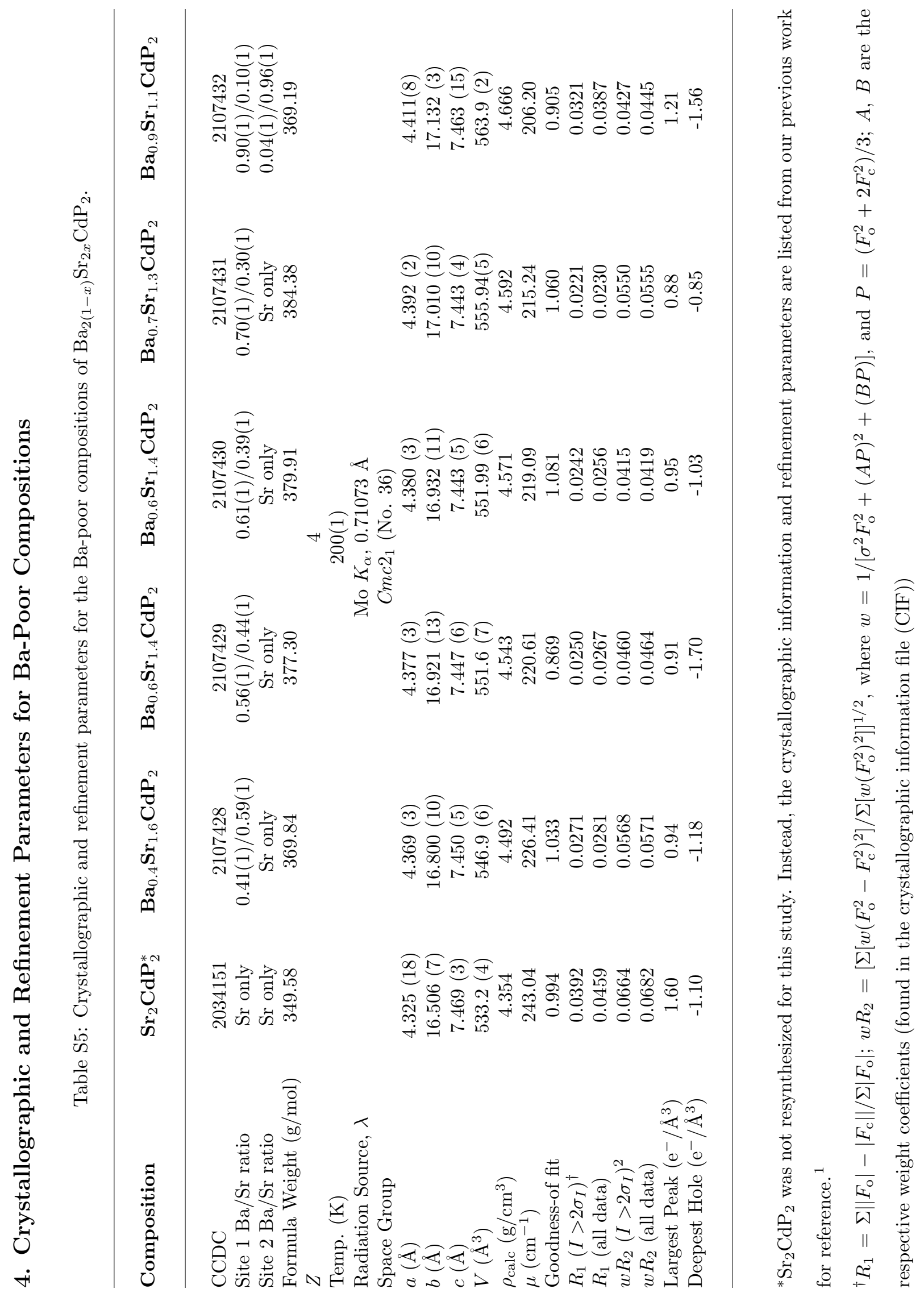




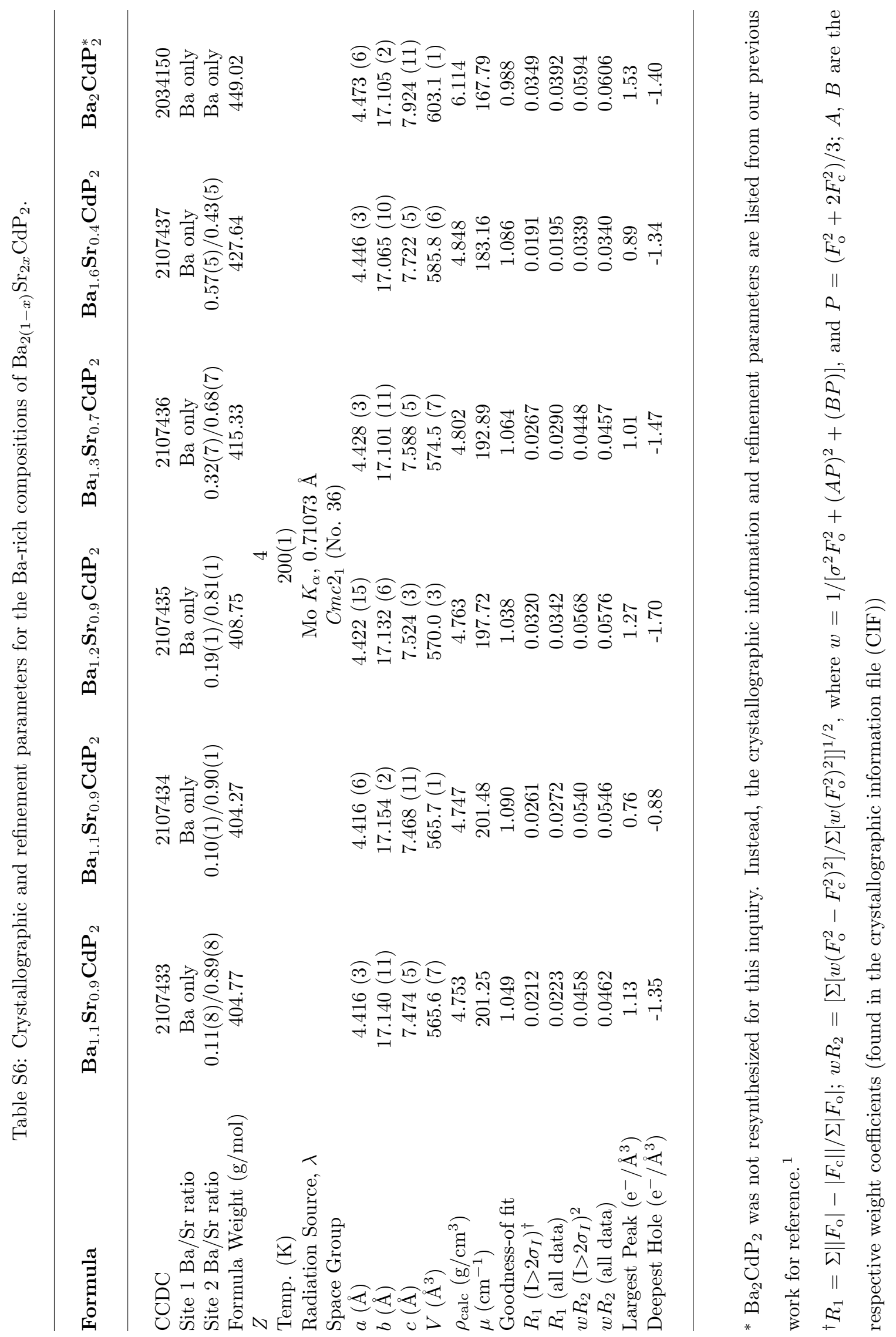




\section{Convergence Test for SQS Used to Calculate Effective Band Structure}

Table S7: Comparison of the electronic structure properties of 100- and 200-atom SQS supercells of $\mathrm{Ba}_{0.4} \mathrm{Sr}_{1.6} \mathrm{CdP}_{2}(x=0.8)$. The band gap $\left(E_{g}\right)$, valence band density-of-states effective mass $\left(m_{\mathrm{DOS}, \mathrm{VB}}^{*}\right)$, and conduction band density-of-states effective mass $\left(m_{\mathrm{DOS}, \mathrm{CB}}^{*}\right)$ are found to be sufficiently converged for the 100 -atom SQS. $E_{g}, m_{\mathrm{DOS}, \mathrm{VB}}^{*}$, and $m_{\mathrm{DOS}, \mathrm{CB}}^{*}$ are calculated from the effective band structures. The DOS effective mass is calculated within a $100 \mathrm{meV}$ energy window from the relevant band edges, following the methodology reported in our previous works. ${ }^{1,2}$

\begin{tabular}{lccc}
\hline size of SQS & $E_{g}(\mathrm{eV})$ & $m_{\mathrm{DOS}, \mathrm{VB}}^{*}\left(m_{\mathrm{e}}\right)$ & $m_{\mathrm{DOS}, \mathrm{CB}}^{*}\left(m_{\mathrm{e}}\right)$ \\
\hline & & & \\
100 & 0.872 & 0.43 & 0.05 \\
200 & 0.872 & 0.31 & 0.06 \\
\hline
\end{tabular}

6. Effective Band Structure of $\mathrm{Ba}_{0.7} \mathrm{Sr}_{1.3} \mathrm{CdP}_{2}(x=0.65)$

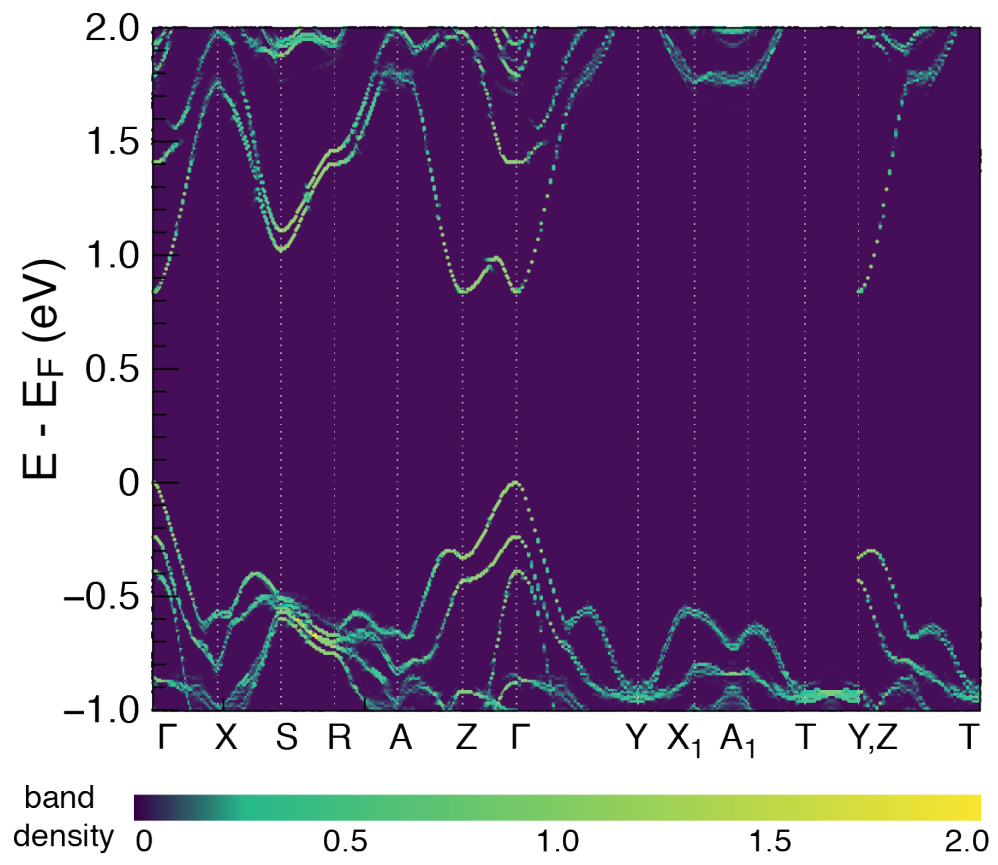

Figure S2: The effective band structure of $\mathrm{Ba}_{0.7} \mathrm{Sr}_{1.3} \mathrm{CdP}_{2}(x=0.65)$ alloy along special $\vec{k}$-point paths of the orthorhombic Brillouin zone. 


\section{Effect of Number of Interpolation Points on the EBS}

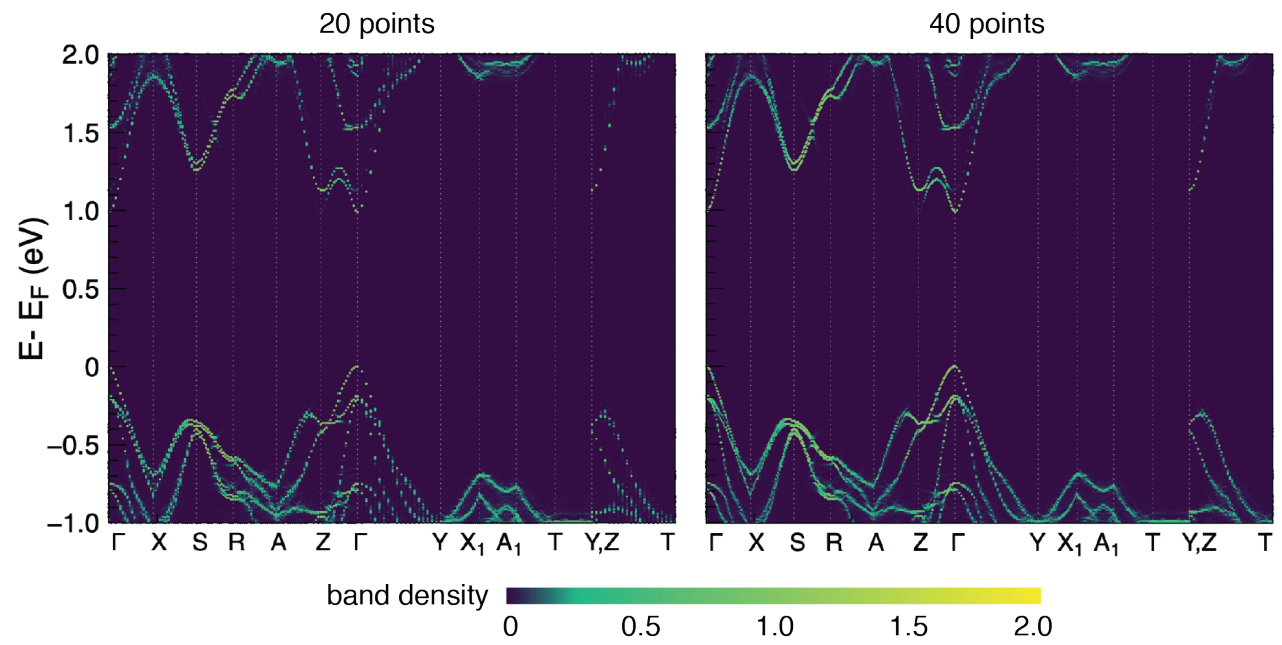

Figure S3: The effective band structure of $\mathrm{Ba}_{0.4} \mathrm{Sr}_{1.6} \mathrm{CdP}_{2}(x=0.8)$ calculated with two different number of interpolation points between each pair of high symmetry $k$-points of the primitive cell.

\section{Atomic Sites in the Defect Supercell}
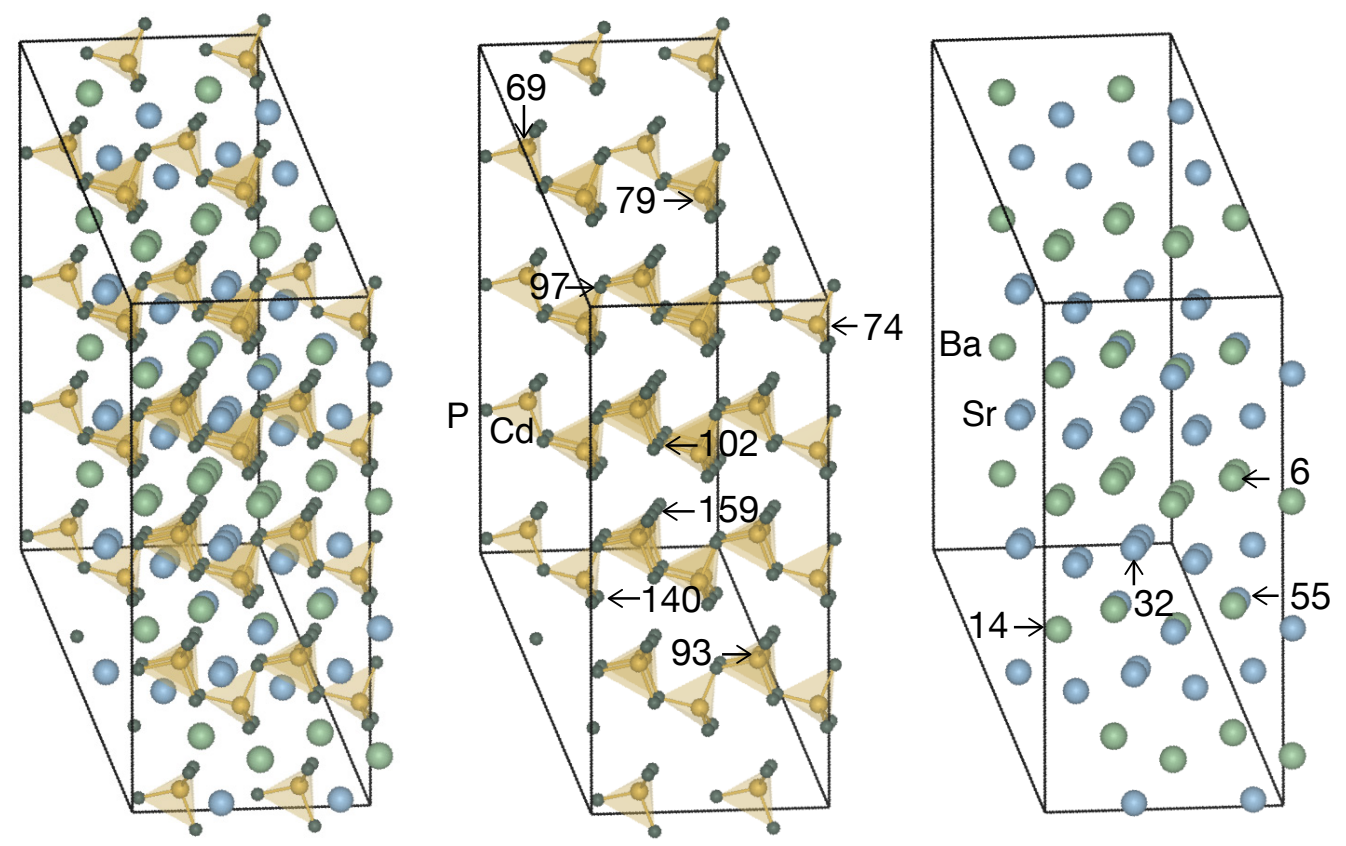

6: $\quad \mathrm{V}_{\mathrm{Ba}(\mathrm{min})}$

14: $\mathrm{V}_{\mathrm{Ba}(\max )}$

32: $\mathrm{V}_{\mathrm{Sr}(\min )}$

55: $V_{S r(\max )}$

69: $\mathrm{V}_{\mathrm{Cd}(\min )}$

74: $V_{\mathrm{Cd}(\max )}$

140: $V_{P(\min )}$

102: $V_{P(\max )}$

97: $\mathrm{Cd}_{\mathrm{P}(\min )}$

159: $\mathrm{Cd}_{\mathrm{P}(\max )}$

93: $P_{C d(\min )}$

79: $P_{C d(\max )}$

Figure S4: Crystal structures of 160-atom supercell of $\mathrm{Ba}_{0.75} \mathrm{Sr}_{1.25} \mathrm{CdP}_{2}(x=0.625)$ used in defect calculations. The atomic indices corresponding to the maximum and minimum $\Delta E_{D, q}$ for each defect in the neutral charge state (Figure 7a in the main text) are labeled. 


\section{Convergence Test for SQS Used in Defect Calculations}

Table S8: Comparison of the electronic structure properties of 40- and 120-atom SQS supercells of $\mathrm{Ba}_{0.75} \mathrm{Sr}_{1.25} \mathrm{CdP}_{2}(x=0.625)$. The band gap $\left(E_{g}\right)$ and energy offset of the conduction bands at $\Gamma$ and $Z\left(\Delta E_{\Gamma-Z}\right)$ are extracted from the EBS. The 40-atom SQS reproduces the electronic properties of the larger 120 -atom SQS, and therefore, can be considered sufficiently converged.

\begin{tabular}{lcc}
\hline \# atoms in SQS & $E_{g}(\mathrm{eV})$ & $\Delta E_{\Gamma-\mathrm{Z}}(\mathrm{eV})$ \\
\hline \multirow{2}{*}{120} & 0.838 & 0.01 \\
& 0.837 & 0.01 \\
\hline
\end{tabular}

\section{Phase Stability of $\mathrm{Ba}_{0.75} \mathrm{Sr}_{1.25} \mathrm{CdP}_{2}$ in Chemical Potential Space}

Table S9: Four-phase equilibrium regions of $\mathrm{Ba}_{0.75} \mathrm{Sr}_{1.25} \mathrm{CdP}_{2}$ in the quaternary Ba-Sr-Cd-P chemical potential space. $\Delta \mu_{X}(X=\mathrm{Sr}, \mathrm{Ba}, \mathrm{Cd}, \mathrm{P})$ are the deviations in the elemental chemical potential from the reference values $\left(\mu_{X}^{0}\right)$, which are listed in Table S10.

\begin{tabular}{|c|c|c|c|c|c|}
\hline Corner & $\begin{array}{c}\Delta \mu_{\mathrm{Sr}} \\
(\mathrm{eV})\end{array}$ & $\begin{array}{c}\Delta \mu_{\mathrm{Ba}} \\
(\mathrm{eV})\end{array}$ & $\begin{array}{c}\Delta \mu_{\mathrm{Cd}} \\
(\mathrm{eV})\end{array}$ & $\begin{array}{l}\Delta \mu_{\mathrm{P}} \\
(\mathrm{eV})\end{array}$ & Phases in equilibrium with $\mathrm{Ba}_{0.75} \mathrm{Sr}_{1.25} \mathrm{CdP}_{2}$ \\
\hline $\mathrm{P}-1$ & -1.040 & -1.239 & -0.086 & -1.634 & $\mathrm{SrCd}_{11-}-\mathrm{SrCd}_{2}-\mathrm{Ba}_{0.6} \mathrm{Sr}_{1.4} \mathrm{CdP}_{2}$ \\
\hline $\mathrm{P}-2$ & -1.040 & -1.139 & -0.086 & -1.672 & $\mathrm{SrCd}_{11}, \mathrm{SrCd}_{2}, \mathrm{Ba}_{0.9} \mathrm{Sr}_{1.1} \mathrm{CdP}_{2}$ \\
\hline $\mathrm{P}-3$ & -1.822 & -1.921 & -0.015 & -0.925 & $\mathrm{SrCd}_{11}, \mathrm{SrCd}_{2} \mathrm{P}_{2}, \mathrm{Ba}_{0.9} \mathrm{Sr}_{1.1} \mathrm{CdP}_{2}$ \\
\hline $\mathrm{P}-4$ & -1.753 & -1.952 & -0.021 & -0.953 & $\mathrm{SrCd}_{2} \mathrm{P}_{2}, \mathrm{SrCd}_{11}, \mathrm{Ba}_{0.6} \mathrm{Sr}_{1.2} \mathrm{CdP}_{2}$ \\
\hline $\mathrm{P}-5$ & -0.367 & -0.566 & -0.049 & -2.125 & $\mathrm{SrP}, \mathrm{SrCd}, \mathrm{Ba}_{0.6} \mathrm{Sr}_{1.2} \mathrm{CdP}_{2}$ \\
\hline $\mathrm{P}-6$ & -0.312 & -0.439 & -0.504 & -2.180 & $\mathrm{Ba}_{4} \mathrm{P}_{3}, \mathrm{SrCd}, \mathrm{SrP}$ \\
\hline $\mathrm{P}-7$ & -0.344 & -0.443 & -0.472 & -2.174 & $\mathrm{Ba}_{4} \mathrm{P}_{3}, \mathrm{SrCd}, \mathrm{Ba}_{0.9} \mathrm{Sr}_{1.1} \mathrm{CdP}_{2}$ \\
\hline $\mathrm{P}-8$ & -1.790 & -1.889 & -0.524 & -0.702 & $\mathrm{Sr}_{3} \mathrm{P}_{4}, \mathrm{SrP}, \mathrm{Ba}_{0.9} \mathrm{Sr}_{1.1} \mathrm{CdP}_{2}$ \\
\hline P-9 & -0.422 & -0.521 & -0.524 & -2.070 & $\mathrm{Ba}_{4} \mathrm{P}_{3}, \mathrm{SrP}, \mathrm{Ba}_{0.9} \mathrm{Sr}_{1.1} \mathrm{CdP}_{2}$ \\
\hline $\mathrm{P}-10$ & -1.790 & -1.989 & -0.449 & -0.702 & $\mathrm{Sr}_{3} \mathrm{P}_{4}, \mathrm{SrP}, \mathrm{Ba}_{0.6} \mathrm{Sr}_{1.4} \mathrm{CdP}_{2}$ \\
\hline $\mathrm{P}-11$ & -2.058 & -2.157 & -0.390 & -0.501 & $\mathrm{Sr}_{3} \mathrm{P}_{4}, \mathrm{SrP}_{2}, \mathrm{Ba}_{0.9} \mathrm{Sr}_{1.1} \mathrm{CdP}_{2}$ \\
\hline $\mathrm{P}-12$ & -2.072 & -2.171 & -0.376 & -0.494 & $\mathrm{SrP}_{3}, \mathrm{SrP}_{2}, \mathrm{Ba}_{0.9} \mathrm{Sr}_{1.1} \mathrm{CdP}_{2}$ \\
\hline $\mathrm{P}-13$ & -2.051 & -2.250 & -0.319 & -0.506 & $\mathrm{Sr}_{3} \mathrm{P}_{4}, \mathrm{SrCd}_{2} \mathrm{P}_{2}, \mathrm{Ba}_{0.6} \mathrm{Sr}_{1.4} \mathrm{CdP}_{2}$ \\
\hline $\mathrm{P}-14$ & -2.058 & -2.250 & -0.320 & -0.501 & $\mathrm{Sr}_{3} \mathrm{P}_{4}, \mathrm{SrCd}_{2} \mathrm{P}_{2}, \mathrm{SrP}_{2}$ \\
\hline $\mathrm{P}-15$ & -2.072 & -2.245 & -0.320 & -0.494 & $\mathrm{SrP}_{3}, \mathrm{SrCd}_{2} \mathrm{P}_{2}, \mathrm{SrP}_{2}$ \\
\hline $\mathrm{P}-16$ & -2.120 & -2.219 & -0.313 & -0.478 & $\mathrm{SrP}_{3}, \mathrm{SrCd}_{2} \mathrm{P}_{2}, \mathrm{Ba}_{0.9} \mathrm{Sr}_{1.1} \mathrm{CdP}_{2}$ \\
\hline $\mathrm{P}-17$ & -0.421 & -0.620 & -0.395 & -2.098 & $\mathrm{SrCd}, \mathrm{SrCd}_{2}, \mathrm{Ba}_{0.6} \mathrm{Sr}_{1.4} \mathrm{CdP}_{2}$ \\
\hline $\mathrm{P}-18$ & -0.421 & -0.520 & -0.395 & -2.136 & $\mathrm{SrCd}, \mathrm{SrCd}_{2}, \mathrm{Ba}_{0.9} \mathrm{Sr}_{1.1} \mathrm{CdP}_{2}$ \\
\hline
\end{tabular}




\section{Reference Elemental Chemical Potentials}

Table S10: Reference elemental chemical potentials.

\begin{tabular}{ll}
\hline Element & $\mu^{0}(\mathrm{eV})$ \\
\hline & \\
$\mathrm{Sr}$ & -4.11 \\
$\mathrm{Ba}$ & -1.83 \\
$\mathrm{Cd}$ & -0.88 \\
$\mathrm{P}$ & -5.11 \\
\hline
\end{tabular}

\section{References}

(1) Balvanz, A.; Qu, J.; Baranets, S.; Ertekin, E.; Gorai, P.; Bobev, S. New n-Type Zintl Phases for Thermoelectrics: Discovery, Structural Characterization, and Band Engineering of the Compounds $A_{2} \mathrm{CdP}_{2}$ ( $A=$ Sr, Ba, Eu). Chem. Mater. 2020, 32, 10697-10707.

(2) Yan, J.; Gorai, P.; Ortiz, B.; Miller, S.; Barnett, S. A.; Mason, T.; Stevanović, V.; Toberer, E. S. Material Descriptors for Predicting Thermoelectric Performance. Energy Environ. Sci. 2015, 8, 983. 